\title{
Structure and evolution of intense austral cut-off lows
}

Article

Accepted Version

Pinheiro, H., Gan, M. and Hodges, K. (2021) Structure and evolution of intense austral cut-off lows. Quarterly Journal of the Royal Meteorological Society, 147 (743). pp. 1-20. ISSN 1477-870X doi: https://doi.org/10.1002/qj.3900 Available at https://centaur.reading.ac.uk/91776/

It is advisable to refer to the publisher's version if you intend to cite from the work. See Guidance on citing.

To link to this article DOI: http://dx.doi.org/10.1002/qj.3900

Publisher: Royal Meteorological Society

All outputs in CentAUR are protected by Intellectual Property Rights law, including copyright law. Copyright and IPR is retained by the creators or other copyright holders. Terms and conditions for use of this material are defined in the End User Agreement.

\section{www.reading.ac.uk/centaur}

\section{CentAUR}

Central Archive at the University of Reading

Reading's research outputs online 


\section{$1 \quad$ Structure and evolution of intense austral Cut-off Lows}

2

3

4

Henri Pinheiro ${ }^{1}$, Manoel Gan ${ }^{1}$, and Kevin Hodges ${ }^{2}$

5

$6{ }^{1}$ Center for Weather Forecast and Climate Studies, National Institute for Space

7 Research, Sao Jose dos Campos, SP, Brazil

$8 \quad{ }^{2}$ Department of Meteorology, The University of Reading, Reading, United Kingdom

9

10

11

12

13

14

15

16

17

18

19

20 Corresponding author: henrirpinheiro@gmail.com

21 Telephone Number: +55 12 3208-6000

22 ORCID: 0000-0003-4363-3206 
24 This study examines the three-dimensional structure and evolution of the 200 most intense Cut-off Lows (COLs) in the Southern Hemisphere (SH). This is done using feature tracking and cyclone-centred compositing based on the ERA-Interim reanalysis.

27 Composites confirm the existence of a well-defined tropospheric moist cold core co28 located with warm dry air in the lower stratosphere. Such cores are surrounded by 29 regions of strong temperature gradients (frontal zones) which move downstream 30 throughout the life cycle. The stratospheric air intrusion into the troposphere is identified in vertical cross-sections of potential vorticity and ozone, a process referred to as tropopause folding. Precipitation occurs ahead of the COLs because of the low (high)-level convergence (divergence) and strong upward motion. The maximum 34 precipitation is observed during decay, indicating a possible link between COLs and surface cyclones. Composites conditioned on relative vorticity and precipitable water suggest these variables may be related to precipitation. The COLs exhibit a westward

37 tilt during their early stages but they change to a barotropic state in the mature stage.

38 Finally, the main characteristics of the COLs are summarized by categories which 39 discriminate different intensities, indicating there are differences in the structure of 40 COLs with consequences for precipitation. These efforts aim to provide new insights 41 into the development of COLs in the SH which could aid in identifying and forecasting 42 their various types and associated precipitation patterns.

43 Keywords: Cut-off Lows; Composites, Structure; Life Cycle, Southern Hemisphere. 


\section{Introduction}

48 Cut-off Low (COL) pressure systems are upper-tropospheric cold lows that form when a mid-latitude upper-tropospheric trough deepens equatorward (Palmén 1949; Palmén and Newton 1969). Typical COLs are short-lived and quasi-stationary systems with irregular trajectories, thereby posing a great challenge for their prediction even with modern 52 forecasting systems (Bozkurt et al. 2016; Muofhe et al. 2020). The statistics of COLs such as their mean preferred locations are extensively documented in studies for the Northern Hemisphere (NH, Price and Vaughan 1992; Nieto et al. 2005; 2008) and Southern Hemisphere (SH; Fuenzalida et al. 2005; Reboita et al. 2010; Pinheiro et al. 2017). Other studies have investigated the key structural features of COLs (Palmén 1949; Hoskins et al. 1985; Qi et al. 1999; Garreaud and Fuenzalida 2007; Satyamurty and Seluchi 2007; Godoy et al. 2011; Ndarana et al. 2020) but have focused on specific regions or individual events, which may be subject to uncertainty due to the system variability. Therefore, the average characteristics related to the structure and evolution of COLs have had less attention, in particular in the SH.

There are many reasons why it is important to study COLs, one of which is related to precipitation and its impact on human activities. For example, COLs are associated with heavy rainfall events and severe flooding in southern Europe and northern Africa (Knippertz and Martin 2005; Delgado et al. 2007; Llasat et al. 2007; Nieto et al. 2008). In the Southern Hemisphere, COLs are found to account for about half of the total 67 precipitation in southeast Australia (Pook et al. 2006), producing more of the heavier rainfall and flood events in coastal areas (Risbey et al. 2009). They are often associated with heavy precipitation on the Eastern Cape coast (South Africa; Singleton and Reason 2006) and anomalously high precipitation may occasionally occur in arid regions, as observed in the Namib and Kalahari Deserts in southern Africa (Muller et al. 2008) and 
the Atacama Desert in South America (Bozkurt et al. 2016; Reboita and Veiga 2017).

73 Additionally, COLs play an important role in the stratosphere-troposphere exchange

74 (STE) (Price and Vaughan 1993), which can occasionally result in abrupt changes in the ozone concentration at high elevation locations (Rondanelli et al. 2002).

Early studies have documented how COLs present well-defined and asymmetrical cloud and precipitation structures where deep convective clouds are typically located on the eastern edge of the vortex, contrasting with relatively dry air on the upstream side (Griffiths et al. 1998; Singleton and Reason 2006; Satyamurty and Seluchi 2007). However, other studies have shown that convective clouds can be seen to the west of the vortex for COLs in the Mediterranean Region (Delgado et al. 2007; Nieto et al. 2008), suggesting there may be regional differences regarding the cloud and precipitation patterns or there are differences in how COLs are defined in each region and study. Differences in precipitation are also evident with respect to the lifecycle stage. Although Delgado et al. (2007) showed that the probability of heavy precipitation decreases considerably after the peak intensity in the Mediterranean COLs, other studies have demonstrated that most of the precipitation in COLs that affect South America occur from the mature to the decay stages (Satyamurty and Seluchi 2007; Godoy et al. 2011; Bozkurt et al. 2016).

Some of the characteristics of COLs that occur in the NH, but particularly in the Iberian Peninsula and Mediterranean regions, were identified and conceptualized by Nieto et al. (2005) and Llasat et al. (2007). The study of Nieto et al. (2005) proposed a conceptual model of COLs that involves four stages: the upper-level trough, tear-off, cut-off and

94 final stage. These stages represent an idealized view of how key meteorological fields vary through the COL lifecycle. However, this structural model appears not to address some crucial aspects, such as the precipitation and how it varies at each stage. It is also 
97 unknown if the Nieto's conceptual model is relevant for COLs in the SH. Hence, a more comprehensive analysis of these systems is needed.

The understanding of the structure and evolution of COLs is one of the keys to understand their associated cloud and precipitation features. There have been several

101 studies focusing on mid-upper level COLs in both hemispheres, but the literature is

102 limited concerning the vertical structure of COLs and its relation with impacts such as 103 precipitation. Scherhag (1939) stated that COLs are strictly related to depressions at 104 high levels with no surface cyclone. Indeed, COLs are stronger in the upper troposphere and weaken toward the surface, and it is not uncommon to find an anti-cyclonic 106 circulation at low levels (Kuo 1949). However, there are situations in which the vortex 107 deepens downward, leading to surface cyclogenesis (Gan and Rao 1996; Mikyfunatsu 108 2004). Thus it is plausible that the vertical depth (extent) is highly variable in COLs and crucial to determining the cloud and precipitation properties, as earlier suggested by

110 Frank (1970) and demonstrated by the findings of Porcù et al. (2007). Porcù et al.

111 (2007) found that deep COLs in the Mediterranean Region are associated with high 112 rainfall rates affecting relatively large areas, while shallow COLs (defined as the 113 vortices confined to high levels) do not often produce precipitation.

114 There have been several regional case studies investigating the structure of COLs 115 (Palmén 1949; Hoskins et al. 1985; Price and Vaughan 1993; Satyamurty and Seluchi 116 2007; Godoy et al. 2011). The pioneering study of Palmén (1949) showed that COLs 117 have a quasi-barotropic structure with a symmetric circulation at high levels, where 118 maximum winds occur at about $200 \mathrm{hPa}$ and intersect with the tropopause region. Other 119 studies have described the structural key features using observations derived from 120 reanalysis datasets (Mikyfunatsu 2004; Nieto et al. 2008; Ndarana et al. 2020) or 121 numerical model outputs (Garreaud and Fuenzalida 2007; Bozkurt et al. 2016). Several 
122 studies have used Potential Vorticity (PV) as a tool to identify and analyse the

123 dynamical evolution of COLs and the tropopause folding. This process contributes to

124 the transfer of high-PV air from the polar stratospheric reservoir to the subtropical

125 troposphere. There are numerous case studies (Palmén 1949; Hoskins et al., 1985; Bell

126 and Bosart 1993) describing the thermal structural properties of COLs, showing the

127 presence of a well-defined cold core in the middle and upper troposphere with a

128 stratospheric warm core aloft. This characteristic contributes to increased atmospheric

129 instability, particularly if the system moves over warm moist surfaces (Kousky and Gan

130 1981; Nieto et al. 2005).

131 It is well-known that COLs are characterised by strong baroclinicity in the upper layers

132 of the troposphere, as documented in several studies (Palmén 1949; Shapiro 1970;

133 Hoskins 1971). An attempt to identify the baroclinic zones associated with the COLs

134 that occur in the Mediterranean region was carried out by Sabo (1992). The study of

135 Sabo (1992) used the thermal frontal parameter (TFP; Clarke and Renard 1966) and

136 found the existence of two baroclinic zones, one in front of the COL centre (i.e. east of

137 the geopotential minimum) which is connected with the frontal cloud band, and the

138 other behind the COL centre associated with a baroclinic boundary. More recently,

139 Nieto et al. (2005) used the TFP as a conceptual criterion for identifying COLs in the

$140 \mathrm{NH}$ (see step 3 of their methodology). According to these studies, the main baroclinic

141 zone is typically found downstream of the trough axis, where the TFP values are higher

142 than those located in the COL centre.

143 Most conceptual models and theories of COLs have essentially been developed through

144 case studies (Palmén 1949; Hoskins et al. 1985; Nieto et al. 2005; Llasat et al. 2007),

145 providing a valuable framework to understand the dynamical evolution of COLs.

146 However, limited sample sizes may bias the conceptual models to specific cases. Hence, 
147 basing a conceptual model on a larger sample size may provide improved insights into

148 COL behaviour. Since mostMost conceptual models have been built on evidence-based

149 case studies for COLs in the $\mathrm{NH}$, where their properties may differ from those

150 associated with SH COLs (because of the association with the zonal asymmetries in

151 topography, land-sea contrast, and seasonal variation of jet stream).-and motivated

152 Motivated by the limited number of studies on the structure of COLs in the SH, and 153 hypothesizing that SH COLs may be different from NH COLs, it is therefore important

154 to identify the average properties presented by typical austral COLs that may be

155 considered as features in conceptual models of SH COLs.

156 The aim of this study is to provide a comprehensive analysis of the mean structure and

157 evolution of the typical COLs observed in the SH. A compositing methodology is used

158 to analyse the key features of COLs through horizontal and vertical composites of 159 several fields relevant to the structure of COLs. Given the differences in the COL 160 characteristics observed from case to case, the main questions to be addressed in this 161 study are the following:

162 1) What is the typical structure and lifecycle of SH subtropical COLs?

163 2) How is the precipitation related to the magnitude of moisture and intensity of the COLs observed in the SH?

165 The focus of this paper is on the extreme austral COLs obtained as the 200 most intense systems (> 98th percentile) identified in reanalysis data during a 36-year period (19792014). This analysis goes beyond the scope of earlier studies, making results more

168 statistically robust and providing a guiding framework for the development of 169 conceptual models for COLs. 
170 The methods used to track and create composite fields of COLs are described in Section

171 2. The spatial statistics for all COLs and the strongest COLs are presented in Section 3.

172 In Section 4 we describe the main structural features of COLs through horizontal and

173 vertical composite fields and provide further insights into the COL structure in the

174 Supplementary Material. Section 5 focuses on the composites of the life cycle of COLs.

175 Section 6 examines how precipitation varies with respect to the intensity of COLs and 176 their moisture content. Finally, two conceptual models are proposed in Section 7 to

177 explain the effect of the intensity on the vertical structure of COLs and their

178 precipitation, together with discussion of the results and concluding remarks.

1792 Data and analysis methodologies

$180 \quad 2.1$ ERA-Interim Reanalysis

181 The main data used in this study comes from the European Centre for Medium-Range

182 Weather Forecast (ECMWF) ERA-Interim (ERAI) Reanalysis (Dee et al. 2011), which

183 is used to provide a detailed view of the structure and evolution of SH COLs. In the

184 absence of any truth to compare with, this reanalysis is in good agreement with other

185 contemporary reanalyses in respect of COL identification and distribution in the SH

186 (Pinheiro et al. 2020). ERAI has been produced by ECMWF using a spectral model

187 with TL255 horizontal resolution ( $~ 80 \mathrm{~km}-)$ and 60 vertical hybrid levels with the model 188 top at $0.1 \mathrm{hPa}$. A 4D-Var data assimilation scheme is used to assimilate the diverse 189 observations from satellite and terrestrial data. For the surface boundary conditions, the

190 Sea Surface Temperature (SST) and Sea Ice Concentration (SIC) are prescribed using

191 different SST and SIC products, as described in Dee et al. (2011). The ERAI data was

192 used for the period from 1979 to 2014.

$193 \quad 2.2$ Tracking and compositing methodology 
194 The SH COLs are identified and tracked in two fields (6 hourly) independently as

195 features with minima below $-1.0 \times 10^{-5} \mathrm{~s}^{-1}$ for filtered $300-\mathrm{hPa}$ relative vorticity $\left(\xi_{300}\right)$

196 and -50 geopotential meters (gpm) for filtered 300-hPa geopotential anomaly $\left(Z_{300}^{\prime}\right)$.

197 The $Z_{300}^{\prime}$ is obtained by removing the zonal mean from geopotential data $\left(Z_{300}\right)$ in order

198 to facilitate the identification (Pinheiro et al. 2019). The reason for the use of both $\xi_{300}$

199 and $Z_{300}^{\prime}$ is to give a generic view of the structure of the SH COLs. Before the

200 identification and tracking, the data are spectrally truncated to T42 for vorticity, as this

201 is a very noisy field, and T63 for geopotential which is a smoother field. The respective

202 minima are first identified at each time step on the grid and then refined using an

203 interpolation and minimization method (Hodges, 1995). The tracking is performed by

204 first initializing a set of tracks from the identified 'feature points' using a nearest

205 neighbour approach and then applying an optimization of a cost function for track

206 smoothness which is subject to adaptative constraints appropriate to the type of motion

207 for the COLs, as discussed in Pinheiro et al. (2019). A post tracking filtering with

208 respect to the $300-\mathrm{hPa}$ horizontal wind components $\left(U_{300}, V_{300}\right)$ is used to avoid open

209 troughs in the analysis. Four offset points located at $5^{\circ}$ (geodesic distance) from the

210 tracked minima are sampled along the tracks in four directions relative to the centre,

211 which are $0^{\circ}\left(U_{300}>0\right), 90^{\circ}\left(V_{300}<0\right), 180^{\circ}\left(U_{300}<0\right)$, and $270^{\circ}\left(V_{300}>0\right)$ relative to

212 North. Given the different choice of methods derived from different variable

213 combinations, the method described above has been found effective for COL

214 identification (Pinheiro et al. 2019) as the mentioned scheme detects the largest number

215 of COLs generally seen in geopotential maps. Only the tracks that reach $40^{\circ} \mathrm{S}$ or have

216 their genesis north of $40^{\circ} \mathrm{S}$ and last at least $24 \mathrm{~h}$ are included. To discard tropical

217 systems, tracks that occur north of $15^{\circ} \mathrm{S}$ are excluded. 
218 A compositing methodology that has previously been applied to tropical and 219 extratropical cyclones (Bengtsson et al. 2009; Catto et al. 2010; Dacre et al. 2012; 220 Hawcroft et al. 2016) is applied here to obtain the composite structure and evolution of

221 COLs. The focus is on extreme COLs obtained from the 200 most intense systems that 222 can be found in both $\xi_{300}$ and $Z_{300}^{\prime}$. The identically same COLs are identified by matching the tracks from the vorticity and geopotential that have a mean separation distances less than four degrees and that overlap in time by at least $50 \%$ of their track points. For the composite procedure, each COL is centred on the time when the $\xi_{300}$ and $Z_{300}^{+}$-lowest minimuma areis found along each track. Then, single level fields (such as precipitation) or multi-level fields (such as winds) are extracted on to a radial grid with 228 maximum radius of $15^{\circ}$ centred on the COL centres $(1 \underline{5}$ degree $\sim 1500 \mathrm{~km})$, which is suitable for capturing the synoptic features of COLs. Since COLs move preferentially eastward and we aim to examine the horizontal tilt, composites are not rotated to the

231 direction of propagation prior to compositing (Catto et al. 2010). This allows us to view 232 the COL horizontal tilt during its development referred to cardinal points relative to 233 North $\left(0^{\circ}\right)$. The vertical tilt is obtained as the (geodesic) angle between the $\xi_{300}$ tracked 234 minima and the corresponding vorticity minima at a number of levels from $300 \mathrm{hPa}$ 235 down to $1000 \mathrm{hPa}$, similar to that performed by Bengtsson et al. (2009) for extratropical 236 cyclones except the search is performed downwards.

\section{$237 \quad 3$ Spatial statistics for all and the strongest Cut-off Lows}

238 Before presenting the results for the structure and evolution of COLs, the hemispheric 239 COL climatology is shown in Figure 1 as the track density computed using spherical 240 kernel estimators (Hodges 1996) using all identified matched COLs (total number is 24111,542 tracks) and for the 200 most intense COLs that match for a 36-year period. The 242 climatological track density shows a distribution very similar to that obtained in earlier 
studies of mid-upper level COLs (Fuenzalida et al. 2005; Reboita et al. 2010; Ndarana

244 and Waugh 2010; Favre et al. 2012; Pinheiro et al. 2017) where the highest values are

245 found in the vicinity of the continents. For the strongest COLs, in particular, the track

246 density maxima shift to more poleward latitudes, being located on the poleward side of

247 the main region of the track density. This is the case for the strongest COLs located in

248 the eastern Indian Ocean, southeast Australia, and southern Africa, where values exceed

2490.3 units, coinciding with the location of large values of mean intensity of COLs (see

250 Fig. 5 of Pinheiro et al. 2017). The strongest COLs that are located at more equatorward

251 latitudes in the SH occur off the west coast of South America (values up to 0.4) where

252 such events frequently affect areas of northern Chile and southern Peru (Garreaud and

253 Fuenzalida 2007; Bozkurt et al. 2016; Reyers and Shao 2019). However, in the western

254 Pacific where the climatological track density is relatively large, the frequency for the strongest COLs is quite small. In this sector, the high COL activity is partly associated

with the high-frequency of blocking (Trenberth and Mo 1985; Marques and Rao 2000)

which may differ from the structure of typical COLs, as described by Palmén (1949).

258 According to Hoskins et al. (1985), many COLs can develop in association with

259 blocking highs as part of the same phenomenon, which can be distinguished by the sign

260 of the isentropic PV anomaly.

\section{Composites of Cut-off Low structure}

262 The main synoptic-scale features of COLs that have been described in case studies and

263 conceptual models are compared with the composites of the 200 most intense COLs

264 from the ERAI reanalysis. The structure of COLs is described through different 265 variables and pressure levels using the identical systems identified in both $\xi_{300}$ and $266 Z_{300}^{\prime}$. Although the analysis is based on the $\xi_{300}$ and $Z_{300}^{\prime}$ COLs, composites are 267 extracted from the time of maximum intensity in $\xi_{300}$ for each COL (see Fig. 1 for the 
268 locations of the maximum intensity in each COL), so the discussion will be in terms of the relative vorticity. The upper-level features of SH COLs will be presented in Section 4.1, followed by an examination of the vertical features in Section 4.2.

\subsection{Upper-level features}

272 In this section the key upper-level features of the strongest COLs are examined at 273 maximum intensity (lowest minima) through different horizontal fields. The composite 274 of the zonal component of the 300-hPa wind speed (Fig. 2a) exhibits a north-south asymmetry where the highest values occur to the north/northeast (wind speeds reaching $50 \mathrm{~m} / \mathrm{s}$ ) as a result of meridional divergence of vorticity advection (Ndarana et al. 2020), contrasting with relatively low easterly winds to the south associated with the vortex detachment. According to Ndarana et al. (2020), the vorticity advection convergence decelerates the flow and facilitates the formation of a split jet structure. The meridional wind component (Fig. 2b) demonstrates there is much more symmetry with respect to the west-east direction with maximum values ranging from 40 to $50 \mathrm{~m} / \mathrm{s}$. The spatial pattern of the absolute wind speed shows two maxima with a discrete westward tilt: the stronger centre occurs in the top right-hand quadrant of the composite COL

284 (northeastern sector); the other centre is located to the west of the COL centre with a smaller extent than the former. However, the wind speed near the tropopause varies widely with the life cycle (see Fig. 5) due to the kinetic energy which is dispersed from upstream by the ageostrophic flux (Gan and Piva 2013). A prominent feature of the COLs is their high-PV anomaly (Fig. 2a-b) which stretches into a narrow streamer equatorward and becomes an isolated PV contour. This process is associated with tropopause folds and downward transport of ozone (see Fig. S2 in the Supplementary

291 Material).One of the main distinguishing features of COLs described in previous conceptual models (Nieto et al. 2005; Llasat et al. 2007) is the cold air in the mid-upper 
troposphere. Some studies described in the literature have shown that the cold air

294 associated with a COL is superimposed on warmer air in the lower stratosphere,

295 characterising a thermal dipole pattern (Palmén 1949; Griffiths et al. 1998; Campetella

296 and Possia 2007; Satyamurty and Seluchi 2007). To identify the approximate position of

297 the cold and warm cores within COLs, the zonal temperature anomaly was examined at

298 different pressure levels (not shown), this suggests that the cold (warm) anomalies cover

299 a deep layer in the troposphere (stratosphere). Figure 2c-d shows the anomalous

300 temperature and TFP (Clarke and Renard 1966) at 400 and $200 \mathrm{hPa}$ which are the two

301 closest levels to the upper-level COLs that can ideally be identified as the cold and

302 warm regions, respectively. It is apparent that the horizontal location of the tropospheric

303 cold anomalies coincides fairly well with that of the stratospheric warm anomalies

304 above. The TFP is used here to identify the baroclinic zones within the COLs since it

305 takes into account the changes of temperature in the direction of the temperature

306 gradient. This exhibits the maxima in the top left-hand quadrant for the composites,

307 with cold and warm cores associated with correspondingly strong horizontal

308 temperature gradients. We found similar results using earlier lifecycle stages, whereas in

309 the decaying lifecycle stage the maximum TFP occurs on the downstream side of the

$310 \mathrm{COL}$ as shown in the supplementary material (Sections 2 and 5). This is in agreement

311 with the conceptual model of Keyser and Shapiro (1986), where the frontal zones

312 propagate downstream throughout the lifecycle of upper-level eddies. However, there

313 are significant differences from case to case which is smoothed out by the compositing.

314 The standard deviation of TFP (Figures S5 and S6 in the Supplementary Material)

315 shows significant spread which can be attributed to different development scenarios.

$316 \quad 4.2$ Vertical features 
317 We now look at the vertical structure of the composite COLs at maximum intensity for

318 several fields through the West-East (W-E) and South-North (S-N) cross sections. The

$31915^{\circ}$ spherical cap region is extracted for ten pressure levels from $1000 \mathrm{hPa}$ up to 100

$320 \mathrm{hPa}$ using the track point at $300 \mathrm{hPa}$ as the reference. Thus, the vertical tilt of COLs is

321 not taken into account for this specific analysis, but it will be examined later. Vertical

322 composites can be produced using either the vorticity or geopotential minimum at each

323 pressure level to extract the region centred on the minimum referred to each level, but

324 the results are not changed since tilt is minor in the mature stage. The vertical

325 composites are exhibited within a $12^{\circ}$ radius, which is where the synoptic-scale

326 processes dominate. Composites are also produced at a specified offset time relative to

327 the time of maximum intensity of COLs identified in both $\xi_{300}$ and $Z_{300}^{\prime}$, and are

328 discussed later in Section 5.

329 Parameters such as wind speed, geopotential, relative vorticity and PV are conveniently

330 discussed together in order to highlight the typical dynamic structure of COLs. A nearly

331 zonally-symmetric circulation is seen in the middle and upper troposphere for the W-E

332 cross section (Fig. 3a) where the centres of maximum wind speed are located between

333300 and $200 \mathrm{hPa}$ and about $4^{\circ}-6^{\circ}$ west and east of the COL centre, with the eastern

334 centre a little stronger and higher than the western centre. The magnitude of the winds is

335 greatly reduced horizontally and vertically away from the maxima, indicating a region

336 of strong horizontal and vertical wind shear in the upper troposphere. However, the S-N

337 cross section (Fig. 3b) shows an asymmetric circulation with rather strong winds at 250

$338 \mathrm{hPa}$ in the northern part of the COL, associated with the westerly winds, which is

339 consistent with Fig. 2a. Similar results have been observed for a COL in North America

340 by the pioneering study of Palmén (1949), which attributed the asymmetry of the N-S

341 cross section to a lack of observations in the southern United States and Mexico. Further 
342 studies such as Hsieh (1949) and Kelley and Mock (1982) confirmed the asymmetry

343 does exist with stronger winds on the equatorward side of the COL. The results of our

344 study are consistent with previous findings as the relatively weak winds appear on the

345 poleward side of the COL and occur due to the weakening of westerlies and reversal of

346 winds associated with the cut-off process.

347 The full resolution of relative vorticity and geopotential height anomaly (Figure 3a-b)

348 are examined to demonstrate the vertical structure of COLs in terms of intensity. The

349 maximum intensity occurs near the tropopause where the wind (shear) magnitude is

350 maximum, but the values gradually weaken toward the surface, though cyclonic features

351 are still found in the lower troposphere. It can be seen that the largest geopotential

352 anomalies (values lower than $-250 \mathrm{gpm}$ ) coincide in location with the vorticity

353 minimum $\left(-25.0 \times 10^{-5} \mathrm{~s}^{-1}\right)$ in the W-E cross section, but the geopotential minimum is

354 slightly offset from the vorticity minimum in the S-N cross section due to the shear

355 component effect in the upper-level flow (Bell and Keyser 1993). Similar results are

356 obtained if the compositing were performed referencing to the 500-hPa vorticity COLs

357 (not shown). This means the maximum intensity of COLs is expected to be at about 300

$358 \mathrm{hPa}$ even if the tracking were performed at a level other than $300 \mathrm{hPa}$. In general, our

359 results are consistent with previous studies on COLs in subtropical regions, indicating

360 that COLs have the strongest intensities at about the $300 \mathrm{hPa}$ level (Quispe and Avalos

361 2006; Satyamurty and Seluchi 2007). This is different from other types of tropopause

362 vortices such as tropopause tropical vortices (TTV) which have maximum intensities at

363 higher levels (Kousky and Gan 1981; Kelley and Mock 1982) or tropopause polar

364 vortices (TPV, Cavallo and Hakim 2010) which have maximum intensities at lower

365 levels. 
366 Another typical aspect of COLs is the stratosphere-troposphere exchange as a 367 consequence of the tropopause folding, as indicated by the $2.0 \mathrm{PVU}$ surface $(1 \mathrm{PVU}=$ $36810^{-6} \mathrm{~m}^{2} \mathrm{~s}^{-1} \mathrm{~K} \mathrm{~kg}^{-1}$ ) in Figure 3a-b. As the tropopause is lowered, high-PV stratospheric 369 air is drawn down into the troposphere, leading to the formation of isolated stratospheric 370 air cut-off from its origin, the so-called "stratospheric reservoir". This process is often 371 associated with Rossby wave breaking (RWB) events, which in turn are strongly 372 connected to the PV deformation, as discussed in many studies (Hoskins et al. 1985; 373 Wernli and Sprenger 2007; Ndarana and Waugh 2010). The deepest intrusion of PV 374 occurs when the COLs reach their maximum intensity, particularly for the S-N cross 375 section where an abrupt variation is observed on the equatorial side of the COL. For 376 very intense COLs, the tropopause can reach lower levels (e.g. $600 \mathrm{hPa}$ ) as seen in 377 Hoskins et al. (1985) and the Supplementary Material (Fig. S11a). Another consequence 378 of the tropopause folding is the penetration of stratospheric ozone into the troposphere, 379 as similarly observed in mid-latitude cyclones (Knowland et al. 2015). Composites 380 show a clear tongue of ozone rich-air near the COL centre, similar to the PV distribution 381 though the tropopause ozone fold in the COL centre is less pronounced compared to that 382 in the PV. The vertical profile of ozone in COLs is given in the Supplementary Material 383 (Section 2).

384 In Section 4.1, the TFP was used to identify the frontal zones within COLs which are 385 located near the maximum temperature gradients. Here the vertical cross-section of the 386 TFP (Fig. 3c-d) indicates that the baroclinic zones associated with COLs are not 387 restricted to a narrow vertical band but they extend through a deep layer in the 388 atmosphere. Frontal boundaries are clearly seen on the edges of the cold and warm cores in the W-E cross section where the narrow regions between the maximum and 390 minimum TFP denote the largest temperature gradient. In the S-N cross section, a single 
well-defined maximum is found on the northern side of the COL which is consistent

392 with the observed TFP distribution shown in Figure 2c-d.

393 The thermal structure of COLs is also examined in terms of isentropes and isotherms 394 and is shown in Figure 4a-b. In the W-E cross section, the isentropes and isotherms 395 appear to be almost completely symmetric, but the S-N cross section exhibits a 396 discernible asymmetry. The tropospheric cold-core is apparent through the isotherms

397 folding in the COL centre where it is surrounded by warmer air, while the stratospheric 398 warm-core is marked by cooler temperatures in the vortex periphery, which is consistent with Figure 2c-d. Contrary to the temperature profile, the potential temperature increases with height, thus the cold core is depicted by a reversal of the lapse rate in the

401 lower troposphere. The thermal structure represented in the composites agrees fairly 402 well with previous studies (Palmén 1949; Griffiths et al. 1998; Campetella and Possia 403 2007; Satyamurty and Seluchi 2007) though the discontinuity of (potential) temperature

404 typically seen along the tropopause is smoothed out by the compositing process.

405 The relative humidity anomaly (Fig. 4a-b) exhibits a pattern similar to the temperature 406 anomaly but with opposite sign, where an anomalously dry stratospheric air is located 407 above the moist tropospheric air. This pattern increases the relative humidity gradient 408 across the tropopause which may be important to intensify the vortex circulation 409 through radiative cooling, as observed in TPVs (Cavallo and Hakim 2010). The W-E 410 cross section (Fig. 4a) shows that the moisture anomalies slope upward and eastward, 411 while the S-N cross section (Fig. 4b) indicates a downward intrusion of stratospheric 412 dry air into the northern flank of the COLs, which is coherent with the tropopause fold 413 indicated by the 2.0 PVU (Fig. 3b).

414 The analysis of the three-dimensional structure of COLs continues by assessing other 415 parameters such as the vertical velocity and divergence (Fig. 4c-d). Similar to the results 
416 discussed above, the largest contrasts are seen in the W-E cross section (Fig. 4c) since

417 COLs move preferentially eastward as discussed before. This shows that the lower and 418 middle troposphere are dominated by convergence downstream and divergence 419 upstream of the storm centre, and the opposite holds at higher levels. As a consequence 420 of the convergence at low levels, the maximum uplift takes place on the downstream 421 side of COLs at $500-400 \mathrm{hPa}\left(\sim 5^{\circ}\right.$ from the vortex centre $)$ so that the moist air is transported to higher levels by the ascent. On the upstream side, a strong descending branch prevails with vertical velocity values comparable to the ascent region. It is apparent that the ascent and descent regions influence almost the entire troposphere, but the maximum values occur at the level of non-divergence, which is approximately at $500 \mathrm{hPa}$ for the descent and somewhat higher for the ascent.

427 Given the characteristics described above, cloud formation and precipitation are expected to be present (absent) on the downstream (upstream) side of COLs. Also, the western side of a COL is associated with descent and stratospheric intrusions as the result of ageostrophic flow (Kentarchos et al. 1999). The results for the vertical

431 structure of COLs are in close agreement with earlier studies (Mikyfunatsu et al. 2004;

432 Knippertz and Martins 2005; Godoy et al. 2011), and the circulation features are even comparable to those found in TPVs (Cavallo and Hakim 2010), but may differ in TTVs which present cold air descending near the centre and warmer air rising at the periphery

435 (Kousky and Gan 1981).

\section{Composites of Cut-off Low lifecycle}

437 This section focuses on the life cycle of COLs by compositing particular fields offset 438 from the time of maximum intensity. This allows us to verify what occurs before and 439 after the time when COLs reach their maximum intensity, namely, time zero. Several 440 composite fields based on the most intense COLs are produced for different stages of 
441 the COL lifecycle (Figure 5) and discussed on the basis of the four stages described in

442 the conceptual model of Nieto et al. $(2005 ; 2008)$ outlined below, supplemented by the

443 results from this study with the focus on the precipitation:

444 a. Upper-level trough $(-48 \mathrm{~h})$ : the initial stage starts from a cold, mid-latitude upper-tropospheric trough that deepens and tilts westward. This amplifying synoptic wave is associated with a band of precipitation orientated northwestsoutheast that looks like a cold-frontal structure. Most of precipitation does not exceed the rainfall rate of $2.0 \mathrm{~mm} / 6 \mathrm{~h}$.

b. Tear-off $(-24 \mathrm{~h})$ : as the trough deepens equatorward, the northern part of the

c. Cut-off and mature stages $(0$ and +24$)$ : the vortex becomes completely detached from the westerlies as COLs reach their maximum intensity. The intensification can be seen through the increased geopotential gradient and vertical motions. The precipitation rapidly increases during this stage, reaching the peak about $24 \mathrm{~h}$ after the maximum intensity (maximum values are greater than $6.0 \mathrm{~mm} / 6 \mathrm{~h}$ ). The increased precipitation is partly due to the strengthening of the ascent in response to the upper-level divergence just ahead of the system. This occurs when the divergence region superimposes the ascent region. Convective clouds are likely to develop in the vortex centre when the system passes over warm moist surfaces (Kousky and Gan 1981; Nieto et al. 2008) which leads to an increase in surface-based instability. 
d. Decay $(+48 \mathrm{~h})$ : the decaying stage is characterised by a horizontal eastward tilt and a significant warming of the vortex core and surroundings before the vortex dissipates through diabatic effects as suggested by previous studies (Hoskins et al. 1985; Price and Vaughan 1993; Satyamurty and Seluchi 2007) or merges into the large-scale upper-level flow (Simpson 1952; Ramage 1962; Nieto et al. 2008). The last stage is marked by a significant considerable decrease in precipitation.

The life cycle of the SH COLs in terms of the geopotential height (Figure 5) is similar to that described by Nieto et al. (2008) for the NH COLs, except the pressure level shown in the schematic of Nieto et al. (2008) depicts the geopotential field at $200 \mathrm{hPa}$ (see their Fig. 4) while the analyses in our study refers to $300 \mathrm{hPa}$ which is the level that has been found where the maximum intensity of the SH COLs occur (see Fig. 3a-b). During the initial stages of the lifecycle, the composite geopotential height shows an elongated appearance with a westward tilt, while a more symmetric and barotropic structure occurs in the final stages. It is interesting to note that the absolute $Z_{300}$ 480 minimum associated with a cyclonic COL centre is found in the tear-off stage $(\sim 8850$ 481 gpm) which occurs $24 \mathrm{~h}$ before the time of maximum cyclonic $\xi_{300}$. This means there 482 are differences in the COL lifecycle with respect to vorticity and geopotential, and this has not been previously documented. Despite the difference between vorticity and 484 geopotential, the stages outlined in the Nieto's conceptual model and confirmed in this 485 study can be used as a guide for the different stages of the COL development regardless 486 of the region in which the COL occurs.

The precipitation composite (Figure 5a) exhibits an asymmetry and a large variation in time where the peak values occur from mature to decay stages. This agrees with earlier 489 findings that examined the precipitation associated with COLs in South America 
490 (Satyamurty and Seluchi 2007; Godoy et al. 2011; Bozkurt et al. 2016), but contradicts

491 the report of Delgado et al. (2007) who found the maximum precipitation in

492 Mediterranean COLs during the earlier stages, suggesting there may be regional

493 differences among COLs with respect to their properties and life cycle.

494 The evolution of the SH COLs shows that the cold core structure (Figure 5d) is largely

495 modified throughout the life cycle, since the greatest extent occurs between the upper-

496 level and cut-off stages, but it reduces horizontally and vertically in the decay stage

497 (vertical fields are shown in the Supplementary Material). During the initial stages, the

498 largest warming occurs in the rearward region (ridge) due to the upper-level

499 convergence and descending air that are heated by adiabatic compression. However,

500 warm anomalies extend eastward in the final stages which agrees well with previous

501 studies in which cold cores are destroyed by diabatic heating (Hoskins et al. 1985;

502 Sakamoto and Takahashi 2005; Garreaud and Fuenzalida 2007). Since the aspects

503 described above are typical of the strongest COLs and assuming that numerical models

504 are capable to capture these aspects, models could be used in practice to predict the

505 COL evolution.

506 The study of the lifecycle of COLs continues by analysing the vertical tilt (Figure 6).

507 Tilt is determined for different stages before and after the time of maximum intensity in

$508 \xi_{300}$. The maximum vertical tilt is observed at the first step (60h before time zero) and

509 at low levels $(900-700 \mathrm{hPa})$ when the distance between the 900 and $300 \mathrm{hPa}$ centres is

510 slightly higher than $2^{\circ}$. The westward vertical tilt observed during the early stages is

511 typical of baroclinic systems, though this tilt is much less than that found in

512 extratropical cyclones (Bengtsson et al. 2009). The vertical tilt reduces as the system

513 approaches its maximum intensity, when the distances for vorticity centres between

514 lower and upper levels do not exceed $1.0^{\circ}$. Once COLs start decaying, their structure 
515 becomes quasi-barotropic and even exhibit a reverse tilt (i.e. an eastward vertical tilt),

516 particularly at high levels. An important feature is the maximum tilt at lower levels

517 observed particularly in the early and late stages. Similar results were found by Randel

518 and Stanford (1985) for the life cycle of baroclinic waves at austral mid-latitudes.

\section{Relationship of the precipitation to environmental features}

\section{$520 \quad 6.1$ Upper-level forcing and moisture content}

521 This section analyses how environmental aspects affect precipitation such as the

522 intensity of COLs and their moisture content. Figure 7 provides a quantitative analysis

523 of the cumulative precipitation measured along each identified COL with respect to

524 different intensity ranges, expressed in terms of statistical boxplots (or box and whisker

525 diagram). This is obtained by accumulating all values between the first and last track

526 points, computed over a $5^{\circ}$ spherical cap (about $500 \mathrm{~km}$ radius) centred on the $\xi_{300}$

527 minimum. The precipitation is not the instantaneous value, it is the precipitation

528 obtained from the forecast model accumulated over a 6-h period with 12 hour forecast

529 lead time (which prevents the influence of model spinup). On the basis of this result, it

530 is clear that the mean cumulative precipitation is affected by the COL intensity. The

531 median does not change much with intensities less than $-8 \times 10^{-5} \mathrm{~s}^{-1}$, but a

532 significantconsiderable increase is observed for the stronger COLs (intensities smaller than $-8 \times 10^{-5} \mathrm{~s}^{-1}$ ) over those of smaller intensities (greater than $-8 \times 10^{-5} \mathrm{~s}^{-1}$ ). The

534 increase is particularly marked for the 75th percentile, meaning that high rainfall

535 amounts are much more frequent in stronger COLs compared to weaker systems.

536 Extreme outliers are omitted from the analysis due to their large values, which are 537 expected when boxplots are applied to large datasets (Hofmann et al. 2017). Regardless

538 of the definition of 'outside value', a close inspection of the largest cumulative 539 precipitation events (> $100 \mathrm{~mm}$ per event) reveals that these occur for different intensity 
540 types, suggesting that other factors, besides upper-level COL intensity, may be

541 important to trigger extreme precipitation, such as moisture and instability.

542 It is expected that the moisture available in the environment surrounding a COL is 543 important to produce precipitation. To check test this hypothesis, we performed a 544 similar experiment to that of Field and Wood (2007) where they categorized lower 545 tropospheric cyclones into different groups according to their intensity (measured in 546 terms of surface wind speed) and moisture (measured in terms of column-integrated 547 water vapor). In the present study, composites of precipitation for the COLs are 548 produced conditioned on $\xi_{300}$ (increasing from left to right) and precipitable water 549 (increasing from bottom to top) (Figure 8). Mean values of the nine composites were 550 calculated for the $\xi_{300}$ COLs relative to the time of maximum intensity averaged within $5515^{\circ}$ spherical arc radius centred on the $\xi_{300}$ minimum. As expected, the precipitation 552 increases simultaneously with both intensity and moisture. The composite with the 553 greatest intensities and moisture content has the largest area-average accumulated 554 precipitation over the $5^{\circ}$ region $(26.1 \mathrm{~mm})$, which is the sum over the whole lifecycle of 555 all selected storms in the composite. However, for this composite the standard deviation 556 shows significant variation in precipitation among the COLs (see Figure S4 in the 557 Supplementary Material). In contrast, the lowest area-average accumulated precipitation 558 is observed for the composite with the smallest intensities and moisture content, which 559 corresponds to $13.8 \mathrm{~mm}$. The wetter composites seem to be associated with the most 560 widespread precipitation, whereas the drier composites have precipitation occurring 561 over reduced areas. These results suggest that moisture is an important factor 562 controlling the precipitation area, though the intensity seems to influence the magnitude 563 of precipitation in the COLs which may be linked to the strength of the low-level 564 convergence. This hypothesis requires further investigation. 
565 It can be seen that the composite with most precipitation (top right of Figure 8) does not

566 have the deepest geopotential centre, but instead the three driest composites have a

567 deeper centre than the three composites with greatest moisture content. The reason for

568 weaker intensities observed in the COLs with high precipitation may be explained by

569 the modification of the dynamical structure of COLs due to diabatic latent heating

570 which induces an upper-level anticyclonic PV anomaly, as discussed in many studies

571 (Davies and Emanuel 1991; Reed et al. 1992; Stoelinga 1996; Ahmadi-Givi et al. 2004).

572 Composites produced relative to the time of maximum precipitation indicate similar

573 results, these are given in the Supplementary Material (Section 3).

\section{$574 \quad 6.2$ Dependence on vertical structure}

575 Some common characteristics that can be used to develop conceptual models of COLs

576 have been identified in this study, though there seems to be a significant variation

577 among the observed COLs suggesting the existence of different types of upper-level 578 cyclonic vortices differing in terms of their vertical structure and weather-related 579 impacts such as precipitation. Figure 9 summarises the differences in characteristics 580 between two different types of COLs: the first one (Fig. 9b) represents the most intense

581 COLs obtained from the composite of the 200 strongest COLs; the second type (Fig. 9a)

582 represents the average COLs obtained from all identified COLs which corresponds to

583 11,542 COLs. This shows how the intensity of COLs affects their structure. For the time

584 of maximum intensity, composites of the most intense COLs show a deep vertical

585 structure with well-defined cyclones reaching into the lower troposphere. The vertical

586 coupling induces strong upward vertical motion and enhanced low-level moisture

587 convergence, contributing to a large amount of precipitation. It has been suggested that 588 the coupling described above occurs when the low-level thermal advection has initiated 
589 (Deveson et al. 2002) hinting to a possible synergistic interaction between upper and

590 lower level features, though there may be different ways for classifying cyclones (Evans

591 et al. 1994; Sinclair and Revell 2000; Catto 2016). The composites produced using all

592 identified COLs representing the average COLs, show a much shallower structure with

593 a well-defined vortex only at high levels. For this case, the perturbation gradually

594 weakens with decreasing height and is replaced by an anti-cyclonic circulation on the

595 western side at the surface. Reduced precipitation rates are observed for this type of

596 COL because of the weakened vertical motions and decoupling between upper and

597 lower level disturbances.

598 A track matching algorithm was applied to the categories described above in order to

599 determine the vertical depth of the COLs. This is done by searching for corresponding

600 vorticity minima at pressure levels lower than $300 \mathrm{hPa}$, starting by matching the 400-

$601 \mathrm{hPa}$ vorticity tracks and successively for other levels down to the $1000-\mathrm{hPa}$ level or the

602 lowest pressure level where the match is found, using the same criteria and thresholds.

603 This is done by applying a prescribed value for the mean separation distance between

604 tracks (chosen here to be 5 degrees geodesic) which overlaps in time for at least one per

605 cent of the track points. We found that only $19.4 \%$ of the average COLs have a 606 corresponding surface cyclone, but the percentage rises to $65 \%$ when the COLs are the

607 strongest systems. This confirms earlier findings that only a few COLs reach the surface

608 and indicates a possible relation between intensity of COLs and their vertical depth.

\section{Discussion and conclusions}

610 This paper provides the first robust view of the structure, lifecycle and properties of SH

611 COLs including how their precipitation depends on their structure. The present study 612 has concentrated on the analysis of the 200 most intense COLs (> 98th percentile)

613 located in the SH. This provides a better understanding of the behaviour of COLs and a 
614 useful forecasting aid. Although there is no single concept of the structure and evolution

615 of COLs because of their diverse nature, some common characteristics can be identified

616 by the use of a compositing method that allows the identification of key features of the

617 most intense COLs. Some of the well-known features of conceptual models reported in

618 previous studies are found here and presented herein including: the horizontal and

619 vertical circulation structures, the cold and warm cores and their associated baroclinic

620 zones, the STE caused by tropopause folding, and the precipitation characteristics and

621 other related features.

622 The results reported in this study support earlier case studies of COLs that have found a

623 roughly symmetrical circulation at upper levels with maximum winds at about $300 \mathrm{hPa}$,

624 creating a region of strong horizontal and vertical wind shear. The contrast between the

625 cold air in the vortex centre and the relatively warm air at the periphery produces a

626 distinctly frontal structure on the borders of these systems. The sharpest temperature

627 gradients in COLs are found across the edges of the mid-upper tropospheric cold core

628 where the baroclinic zones normally propagate downstream, which agrees well with the

629 conceptual model described by Keyser and Shapiro (1986) for upper-level fronts. The

630 STE is a consequence of the tropopause deformation in COLs where large amounts of 631 stratospheric air are transported into the troposphere. Our results are consistent with

632 earlier reports indicating that the vertical distribution of PV and ozone are significantly

633 modified in strong COLs. In the mature stage, the vortex tropopause is lowered to 634 around 400-500 hPa, although deeper stratospheric intrusion events can sink the 635 tropopause down to 600-700 hPa, as reported in Hoskins et al. (1985) and observed by 636 individual cases in the present study (see the Supplementary Material, Section 5).

637 A key finding of this study, which has not been addressed in previous studies, is that 638 there are clearly distinct differences in the vertical structure of the SH COLs which can 
639 be demonstrated by means of two different types of COLs. The two models of COLs are

640 similar in terms of their spatial distribution of vertical motions and precipitation but

641 they differ with respect to their magnitude. The major difference between the two

642 groups lies in the contrast between a deep COL structure extending down to the surface

643 and a COL confined at mid-upper levels, suggesting that the differences observed in the

644 vertical depth of the COLs are in part a consequence of the precipitation amount. In this

645 sense, inspection of synoptic weather charts and satellite images provide additional

646 evidence that not all COLs have the same structure and precipitation characteristics and

647 that regional perspectives depending on local topography and surface condition features

648 are important.

649 We have for practical reasons restricted the analysis to the COLs that affect subtropical 650 regions. However, similarities and differences between COLs and other tropopause vortex types (including tropical and polar vortices) are not clearly defined. Some of the structural properties of COLs that have been identified in this study can be found as dominant features in TPVs (Cavallo and Hakim 2010), such as the vertical motions

654 where air rises (sinks) east (west) of the vortex centre, and the cold and warm anomalies 655 in the troposphere and stratosphere, respectively. These systems can be linked to RWB events as both are isolated PV anomalies but COLs are located equatorward of the jet stream while TPVs poleward of the jet.

658 In comparison to TTVs, the COLs studied here have properties comparable to those 659 described in previous studies such as a lowered tropopause and a warm anomaly 660 superimposed on a cold anomaly (Frank 1970; Kousky and Gan 1981; Mishra et al. 661 2001). However, the vertical motion in TTVs exhibits a more symmetrical pattern, with 662 sinking air near the vortex centre and rising air at the periphery (Kousky and Gan 1981). 663 Moreover, while Kousky and Gan (1981) observed that the deepest convective clouds 
664 are seen in the direction of the movement which is often westward in tropical latitudes,

665 our results indicate that the largest precipitation occurs in the eastern side of the COLs.

666 Nevertheless, it might be interesting in future studies to contrast the vertical structure

667 and precipitation of all tropopause vortices, for example, using as identification field the

668 potential temperature on the 2.0 PVU surface.

669 It is also useful to point out how the characteristics of the SH COLs studied here (e.g. 670 precipitation) compare with those associated with $\mathrm{NH}$ COLs such as the systems 671 observed in the Iberian Peninsula and Mediterranean regions (Nieto et al. 2005; 2008;

672 Delgado et al. 2007; Porcù et al. 2007). The distribution of precipitation and cloud cover 673 (the last one not shown here) reproduces the main rainfall band located in the leading 674 edge of the COL (east flank) but does not demonstrate the secondary region in the rear 675 edge of the system (west flank), which in turn is associated with a descending branch. 676 We found that the peak precipitation occurs from the mature to decay stages, which is in 677 agreement with earlier studies on COLs that occurred in South America (Satyamurty 678 and Seluchi 2007; Godoy et al. 2011; Bozkurt et al. 2016). However, most of the 679 precipitation is found in earlier stages in NH COLs (Delgado et al. 2007; Nieto et al. 680 2008), and this may be due to regional differences or the way in which a COL is 681 defined. Differences are also seen by comparing COLs with extratropical cyclones 682 (Bengtsson et al. 2009) which produce more precipitation in their intensifying phase. 683 One possible explanation might be that deep COLs and surface cyclones are often part 684 of the same phenomenon in the development of an upper-level precursor prior to the 685 low-level cyclone, as discussed by Mikyfunatsu et al. (2004).

686 Although COLs are often referred to as cold pools due to their well-defined cold-core 687 structure, a general consensus on the location of the cold anomalies is still missing. One 688 particularly important factor in determining the vertical location of the cold core of 
689 COLs may be the dynamical tropopause position which varies depending on factors

690 such as latitude, season and synoptic situation. Our results show that the tropopause

691 intersects in the COL centre inside the cold core (just above the coldest anomalies)

692 within a region of sharp temperature gradient, also called frontal or baroclinic zone.

693 However, there have been many studies on COLs using methods based on criteria that

694 search for cold cores at very high levels (for example, between 200 and $300 \mathrm{hPa}$ ) in

695 both the southern and northern hemispheres (Nieto et al. 2005; Gimeno et al. 2007;

696 Porcù et al. 2007; Reboita et al. 2010; Muñoz et al. 2019) which may result in

697 differences between studies, as recently discussed by Pinheiro et al. (2019).

698 Unfortunately the lack of studies focusing on the structure of COLs and the difference

699 between hemispheres does not allow us to determine whether the difference in

700 interpretation is a result of the regional peculiarities. There is thus a clear need for more

701 observational studies on COLs in both hemispheres to create a better conceptual

702 framework encompassing the variety of the structures presented in COLs and other cold

703 core vortex types.

704 To summarize our current understanding of COLs, a schematic depiction of the

705 structural features is presented in Fig. 10. This schematic is adapted from Llasat et al.

706 (2007), which is based on the typical properties of Mediterranean COLs, and despite the

707 subjective interpretation there is agreement on the vertical profile of COLs since both

708 schematics denote similar features. The most notable difference is the pressure level of

709 the warm anomalies which is considerably lower ( 300 hPa) in Llasat et al. (2007)

710 compared to those of our composite COL. sThe schematic presented in Fig. 10

711 illustrates the dominant mechanisms and key properties of COLs such as their

712 circulation structures, tropopause folding, cold and warm cores and their associated

713 baroclinic zones. This schematic together with results shown in this study may serve as 
714 a reference guide for the diagnosis of the structural and precipitation features in COLs,

715 helping meteorologists understand weather patterns and produce better forecasts.

716 Although we have not specifically addressed the issue of forecasting ability of

717 numerical weather prediction (NWP) models, this is a problem of great scientific

718 importance for understanding problems typically found in predicting COLs. Many

719 studies have demonstrated that diabatic effects play an important role in the

720 development of different vortex types, with radiative cooling and latent heating

721 contributing to strengthening and weakening, respectively (Hoskins et al. 1985; Katzfey

722 and Mcinnes 1996; Sakamoto and Takahashi 2005; Garreaud and Fuenzalida 2007;

723 Cavallo and Hakim 2010). It is therefore fundamental to explore the predictive skill of

724 forecast systems for COL activity, as has been done for extra-tropical and tropical

725 cyclones (Froude et al. 2007; Hodges and Klingaman 2019). Moreover, better

726 predictions require a detailed understanding of the processes that govern the

727 development of COLs, which is beyond the scope of this paper, but will be explored in

728 future research.

729 This work could also be extended to investigate the cloud properties, but the reanalyses

730 are not well constrained in terms of clouds since the cloud cover is not assimilated into

731 the reanalyses, but instead is predicted by a short range forecast using the same

732 atmospheric model used by the ERAI reanalysis. The use of remote sensing data, such

733 as the International Satellite Cloud Climatology Project data set (ISCCP), could be used

734 to more precisely capture the cloud structure of COLs, as has been performed more

735 generally for extratropical cyclones (Field and Wood 2007; Hawcroft et al. 2012; 2017).

736 Finally, despite the contribution of this study to our understanding of COLs, it is unclear

737 whether the results showed herein would be robust enough to represent the structure and

738 evolution of all COLs around the world given the different vortex types. The problem 
concerning the variety of methods used to identify COLs (Pinheiro et al. 2019) raises

740 the question of whether the different criteria are due to the different conceptual models as a result of the interpretation or differences in structure between the COLs in the

742 Northern and Southern Hemispheres. For this reason we believe that further studies are

743 needed to investigate a possible regional dependence of the COL structure and its

744 evolution as COLs may exhibit different characteristics when they occur in different

745 regions, so that it would be possible to better understand how COLs behave in certain

746 regions while also allowing us to adjust the identification methods to particular

747 geographical locations. Another problem of great scientific importance is how large-

748 scale atmospheric modes of variability influence on COL related properties (such as

749 location, intensity and precipitation). This is crucial for understanding the climate and

750 its variation, and more specifically the effect of the large-scale modes of variability on

751 the seasonal and sub-seasonal prediction of COLs.

\section{Acknowledgements}

753 We thank the anonymous reviewers for their constructive comments which have helped

754 to improve the paper. The work was partly sponsored by CNPq (Conselho Nacional de

755 Desenvolvimento Científico e Tecnológico) and CAPES (Coordenação de 756 Aperfeiçoamento de Pessoal de Nível Superior). The University of Reading (England) 757 and National Institute for Space Research (INPE, Brazil) provide support through 758 computing resources. The authors are also grateful to Jorge Conforte for his help in 759 producing a plot. 


\section{References}

770 Ahmadi-Givi, F., Graig, G.C, Plant, R.S. (2004) The dynamics of a midlatitude cyclone

771 with very strong latent-heat release. Quarterly Journal of the Royal Meteorological 772 Society 130(596), 295-323.

773 Bell, G.D. and Bosart, L.F. (1993) A case study diagnosis of the formation of an upper-

774 level cutoff cyclonic circulation over the eastern United States. Monthly Weather

775 Review 121(6), 1635-1655.

776 Bengtsson, L., Hodges, K., Keenlyside, N. (2009) Will extratopical storms intensify in a

777 warmer climate? Journal of Climate 22(9), 2276-2301.

778 Bozkurt, D., Rondanelli, L., Garreaud, R. and Arriagada, A. (2016) Impact of warmer 779 eastern tropical Pacific SST on the March 2015 Atacama floods. Monthly Weather $780 \quad$ Review 144(11), 4441-4460.

781 Campetella, C.M. and Possia, N.E. (2007) Upper-level cut-off lows in southern South

782 America. Meteorology and Atmospheric Physics 96(1-2), 181-191.

783 Catto, J.L, Shaffrey, L.C. and Hodges, K.I. (2010) Can climate models capture the

784 structure of extratropical cyclones? Journal of Climate 23(7), 1621-1635.

785 Catto, J.L. (2016) Extratropical cyclone classification and its use. Reviews of 786 Geosphysics 54(2), 486-520.

787 Cavallo, S.M. and Hakim, G.J. (2010) Composite structure of tropopause polar 788 cyclones. Monthly weather review 138(10), 3840-3857. 
789 Clarke, L.C. and Renard, R.J. (1966) The U.S. Navy numerical frontal analysis scheme:

790 further development and a limited evaluation. Journal of Applied Meteorology 5(6),

$791 \quad 764-777$.

792 Dacre, H.F., Hawcroft, M.K., Stringer, M.A. and Hodges, K.I. (2012) An extratropical

793 cyclone atlas: a tool for illustrating cyclone structure and evolution characteristics.

794 Bulletin of the American Meteorological Society 93(10), 1497-1502.

795 Davis, C.A. and Emanuel, K.A. (1991) Potential vorticity diagnostics of cyclogenesis.

796 Monthly Weather Review 119(8), 1929-1953.

797 Dee, D.P., Uppala, S.M. and Simmons, A.J., Berrisford, P. Poli P et al (2011) The ERAI

798 reanalysis: configuration and performance of the data assimilation system. Quarterly

799 Journal of the Royal Meteorological Society 137(656), 553-597.

800 Delgado, G., Redaño, G., Lorente, J., Nieto, R., Gimeno, L., Ribera, P., Barriopedro, D.,

801 García-Herreras, R. and Serrano, A. (2007) Cloud cover analysis associated to cut-off

802 low pressure systems over Europe using Meteosat imagery. Meteorology and

803 Atmospheric Physics 96(1-2), 141-157.

804 Deveson, A.C.L., Browning, K.A. and Hewson, T.D. (2002) A classification of

805 FASTEX cyclones using a height-attributable quasi-geostrophic vertical-motion

806 diagnostic. Quarterly Journal of the Royal Meteorological Society 128(579), 93-117.

807 Evans, M.S., Keyser, D., Bosart, L.F. and Lackman, G.M. (1994) A satellite-derived

808 classification scheme for rapid maritime cyclogenesis. Monthly Weather Review 122(7),

809 1381-1416.

810 Favre, A., Hewitson, B., Tadross, M., Lennard, C. and Cerezo-Mota, R. (2012)

811 Relationships between cut-off lows and the semiannual and southern oscillations.

812 Climate Dynamics 38(7-8), 1473-1487. 
813 Field, P.R. and Wood, R. (2007) Precipitation and cloud structure in midlatitude

814 cyclones. Journal of Climate 20(2), 233-254.

815 Frank, N.L. (1970) On the energetics of cold lows. In: Symposium on Tropical

816 Meteorology. Proceedings... American Meteorological Society EIV1-EIV6.

817 Froude, L.S., Bengtsson, L., and Hodges, K.I. (2007) The prediction of extratropical

818 storm tracks by the ECMWF and NCEP ensemble prediction systems. Monthly weather

819 review, 135(7), 2545-2567.

820 Fuenzalida, H.A., Sánchez, R. and Garreaud, R.D. (2005) A climatology of cutoff lows

821 in the Southern Hemisphere. Journal of Geophysical Research 110:D18101.

822 Gan, M.C. and Rao, V.B. (1996) Case studies over South America. Meteorological

823 Applications 3, 359-367.

824 Gan, M.A. and dal Piva, E. (2013) Energetics of a Southeastern Pacific cut-off low.

825 Atmospheric Science Letters 14(4), 272-280.

826 Garreaud, R.D. and Fuenzalida, H.A. (2007) The influence of the Andes on cutoff lows:

827 a modeling study. Monthly Weather Review 135(4), 1596-1613.

828 Gimeno, L., Nieto, R. and Trigo, R.M. (2007) Decay of the Northern Hemisphere

829 stratospheric polar vortex and the occurrence of cut-off low systems: An exploratory

830 study. Meteorology and Atmospheric Physics 96(1-2), 21-28.

831 Godoy, A.A., Possia, N.E., Campetella, C.M. and Skabar, Y.G. (2011) A cut-off low in

832 southern South America: dynamic and thermodynamic processes. Revista Brasileira de

833 Meteorologia 26(4), 503-514.

834 Griffiths, M., Reeder, M.J., Low, D.J. and Vincent, R.A. (1998) Observation of a cut-off

835 Low over southern Australia. Quarterly Journal of the Royal Meteorological Society, $836 \quad \mathbf{1 2 4}(548), 1109-1132$. 
837 Hawcroft, M.K., and Shaffrey, L.C., Hodges, K.I. and Dacre, H.F. (2012) How much

838 Northern Hemisphere precipitation is associated with extratropical cyclones?

839 Geophysical Research Letters 39(24), 1-7.

840 Hawcroft, M.K., Shaffrey, L.C., Hodges, K.I. and Dacre, H.F. (2016) Can climate

841 models represent the precipitation associated with extratropical cyclones? Climate

842 Dynamics 47(3-4), 679-695.

843 Hawcroft, M., Dacre, H., Forbes, R., Hodges, K., Shaffrey, L. and Stein, T. (2017)

844 Using satellite and reanalysis data to evaluate the representation of latent heating in

845 extratropical cyclones in a climate model. Climate Dynamics 48(7-8), 2255-2278.

846 Hodges, K.I. (1995) Feature tracking on the unit sphere. Monthly Weather Review

847 123(12), 3458-3465.

848 Hodges, K.I. (1996) Spherical nonparametric estimators applied to the UGAMP model

849 integration for AMIP. Monthly Weather Review 124(12), 2914-2932.

850 Hodges, K.I., and Klingaman, N.P. (2019) Prediction errors of tropical cyclones in the

851 western North Pacific in the Met Office global forecast model. Weather and

852 Forecasting, 34(5), 1189-1209.

853 Hofmann, H., Wickham, H. and Kafadar, K. (2017) Value plots: Boxplots for large

854 data. Journal of Computational and Graphical Statistics 26(3), 469-477.

855 Hoskins, B.J. (1971) Atmospheric frontogenesis models: some solutions. Quarterly

856 Journal of the Royal Meteorological Society 97(412), 139-153.

857 Hoskins, B.J., Mcintyre, M.E. and Robertson, A.W. (1985) On the use and significance

858 of isentropic potential vorticity maps. Quarterly Journal of the Royal Meteorological

859 Society 111(470), 877-946.

860 Hsieh, Y. (1949) An investigation of a selected cold vortex over North America. Journal

861 of Meteorology 6(6), 401-410. 
862 Katzfey, J.J., and Mcinnes, K.L. (1996) GCM simulations of eastern Australian cutoff

863 lows. Journal of climate, 9(10), 2337-2355.

864 Kelley, W.E. and Mock, D.R. (1982) A diagnostic study of upper tropospheric cold lows

865 over the western North Pacific. Monthly Weather Review 110(6), 471-480.

866 Kentarchos, A.S., Roelofs, G.J. and Lelieveld, J. (1999) Model study of a stratospheric

867 intrusion event at lower midlatitudes associated with the development of a cutoff low.

868 Journal of Geophysical Research Atmospheres 104(D1), 1717-1727.

869 Keyser, D. and Shapiro, M.A. (1986) A review of the structure and dynamics of upper-

870 level frontal zones. Monthly Weather Review 114(2), 452-499.

871 Knippertz, P. and Martin, J.E. (2005) Tropical plumes and extreme precipitation in

872 subtropical and tropical West Africa. Quarterly Journal of the Royal Meteorological

873 Society 131(610), 2337-2365.

874 Knowland, K.E., Doherty, R.M. and Hodges, K.I. (2015) The effects of springtime mid-

875 latitude storms on trace gas composition determined from the MACC

876 reanalysis. Atmospheric Chemistry and Physics 15(6), 3605-3628.

877 Kousky, V.E. and Gan, M.A. (1981) Upper tropospheric cyclonic vortices in the

878 subtropical South Atlantic. Tellus 33(6), 538-551.

879 Kuo, H.L. (1949) Dynamic instability of two-dimensional non-divergent flow in a

880 baroclinic atmosphere. Journal of Meteorology 6(2), 105-122.

881 Llasat, M.C., Martín, F., Barrera, A. (2007) From the concept of "Kaltlufttropfen” (cold

882 air pool) to the cut-off low. The case of September 1971 in Spain as an example of their

883 role in heavy rainfalls. Meteorology and Atmospheric physics 96(1-2), 43-60.

884 Marques, R.F.C. and Rao, V.B. (2000) Interannual variations of blockings in the

885 Southern Hemisphere. Journal of Computational Physics 105(D4), 4625-4636. 
886 Mikyfunatsu, B., Gan, M.A. and Caetano, E. (2004) A case study of orographic

887 cyclogenesis over South America. Revista Atmosfera 17(2), 91-113.

888 Mishra, S.K., Rao, V.B. and Gan, M.A. (2001) Structure and evolution of the large-scale

889 flow and an embedded upper-tropospheric cyclonic vortex over Northeast Brazil.

890 Monthly weather review 129(7), 1673-1688.

891 Muller, A., Reason, C.J.C. and Fauchereau, N. (2008) Extreme rainfall in the Namib

892 Desert during late summer 2006 and influences of regional ocean

893 variability. International Journal of Climatology: A Journal of the Royal Meteorological

894 Society 28(8), 1061-1070.

895 Muñoz, C., Schultz, D. and Vaughan, G. (2019) A midlatitude climatology and

896 interannual variability of 200-and 500-hPa cut-off lows. Journal of Climate 33(6),

$897 \quad 2201-2222$.

898 Muofhe, T.P., Chikoore, H., Bopape, M.J., Nethengwe, N. S., Ndarana, T. and Gift, T.R. (2020)

899 Forecasting Intense Cut-Off Lows in South Africa Using the $4.4 \mathrm{Km}$ Unified Model. Preprints.

900 Ndarana, T. and Waugh, D.W. (2010) The link between cut-off lows and Rossby wave

901 breaking in the Southern Hemisphere. Quarterly Journal of the Royal Meteorological

902 Society 136(649), 869-885.

903 Ndarana, T., Rammopo, T.S., Chikoore, H., Barnes, M.A., and Bopape, M.J. (2020) A quasi-

904 geostrophic diagnosis of the zonal flow associated with cut-off lows over South Africa and

905 surrounding oceans. Climate Dynamics, Preprints.

906 Nieto, R., Gimeno, L., de la Torre, L., Ribeira, P., Gallego, D., García-Herrera, R. et al

907 (2005) Climatological features of cutoff low systems in the Northern Hemisphere.

908 Journal of Climate 18(16), 3085-3103. 
909 Nieto, R., Sprenger, M., Wernli, H., Trigo, R.M. and Gimeno, L. (2008) Identification

910 and climatology of cut-off low near the tropopause. Annals of the New York Academy of

911 Sciences 1146(1), 256-290.

912 Palmén, E. (1949) Origin and structure of high-level cyclones south of the maximum

913 westerlies. Tellus 1(1), 22-31.

914 Palmén, E. and Newton, C.W. (1969) Atmospheric circulation systems: their structure

915 and physical interpretation. New York: Academic Press.

916 Pinheiro, H.R., Hodges, K.I., Gan, M.A. and Ferreira, N.J. (2017) A new perspective of

917 the climatological features of upper-level cut-off lows in the Southern

918 Hemisphere. Climate Dynamics 48(1-2), 541-559.

919 Pinheiro, H.R., Hodges, K.I. and Gan, M.A. (2019) Sensitivity of identifying Cut-off

920 Lows in the Southern Hemisphere using multiple criteria: implications for numbers,

921 seasonality and intensity. Climate Dynamics 53(11), 6699-6713.

922 Pinheiro, H.R., Hodges, K.I. and Gan, M.A. (2020) An intercomparison of Cut-off Lows

923 in the subtropical Southern Hemisphere using recent reanalyses: ERA-Interim, NCEP-

924 CFSR, MERRA-2, JRA-55, and JRA-25. Climate Dynamics 54, 777-792.

925 Pook, M.J., Mcintosh, P.C. and Meyers, G.A. (2006) The synoptic decomposition of 926 cool-season rainfall in the southeastern Australian cropping region. Journal of Applied 927 Meteorology and Climatology 45(8), 1156-1170.

928 Porcù, F., Carrassi, A., Medaglia, C.M., Prodi, F. and Mugnai, A. (2007) A study on cut929 off low vertical structure and precipitation in the Mediterranean region. Meteorology 930 and Atmospheric Physics 96(1-2), 121-140.

931 Price, J.D. and Vaughan, G. (1992) Statistical studies of cut-off low systems. Annales

932 Geophysicae 10, 96-102. 
933 Price, J.D. and Vaughan, G. (1993) The potential for stratosphere-troposphere exchange

934 in cut-off low systems. Quarterly Journal of the Royal Meteorological Society 119(510), $935 \quad 343-365$.

936 Qi, L., Leslie, L.M., and Zhao, S.X. (1999) Cut-off low pressure systems over southern 937 Australia: climatology and case study. International Journal of Climatology: A Journal 938 of the Royal Meteorological Society, 19(15), 1633-1649.

939 Quispe, N. and Avalos, G. (2006) Intense snowstorm in the southern mountains of Peru 940 associated to the incursion to the incursion of cut-off low-pressure systems at upper

941 level. In: International Conference on Southern Hemisphere Meteorology and

942 Oceanography (ICSHMO), Foz do Iguaçu-Brazil, 1945-1958.

943 Ramage, C.S. (1962) The subtropical cyclone. Journal of Geophysical Research 67(4), 944 1401-1411.

945 Randel, W.J. and Stanford, J.L. (1985) The observed life cycle of a baroclinic instability. 946 Journal of the Atmospheric Sciences 42(13), 1364-1373.

947 Reboita, M.S., Nieto, R., Gimeno, L., Rocha, R.P., Ambrizzi, T., Garreaud, R. and 948 Kruger, L.F. (2010) Climatological features of cutoff low systems in the Southern 949 Hemisphere. Journal of Geophysical Research: Atmospheres 115, D17104.

950 Reboita, M.S. and Veiga, J.A.P. (2017) Synoptic and energetic analysis of a Cut-off 951 Low that caused precipitation over the Atacama Desert in March, 2015 (Análise sinótica 952 e energética de um VCAN que causou chuva no deserto do atacama em março de 2015) 953 Revista Brasileira de Meteorologia 32(1), 123-139.

954 Reed, R.J., Stoelinga, M.T. and Kuo, Y.H. (1992) A model-aided study of the origin 955 and evolution of the anomalously high potential vorticity in the inner region of a rapidly 956 deepening marine cyclone. Monthly Weather Review 120(6), 893-913. 
957 Reyers, M. and Shao, Y. (2019) Cutoff lows off the coast of the Atacama Desert under 958 present day conditions and in the Last Glacial Maximum. Global and Planetary Change 959102983.

960 Risbey, J.S., Pook, M.J., Mcintosh, P.C., Ummenhofer, C.C. and Meyers, G. (2009)

961 Characteristics and variability of synoptic features associated with cool season rainfall

962 in southeastern Australia. International Journal of Climatology 29(11), 1595-1613.

963 Rondanelli, R., Gallardo, L. and Garreaud, R.D. (2002) Rapid changes in ozone mixing 964 ratios at Cerro Tololo $\left(30^{\circ} 10^{\circ} \mathrm{S}, 70^{\circ} 48^{\circ} \mathrm{W}, 2200 \mathrm{~m}\right)$ in connection with cut-off lows and 965 deep troughs. Journal of Geophysical 107, D23.

966 Sabo, P. (1992) Application of the thermal front parameter to baroclinic zones around 967 cut-off lows. Meteorology and Atmospheric Physics 47(2-4), 107-115.

968 Sakamoto, K., and Takahashi, M. (2005) Cut off and weakening processes of an upper 969 cold low. Journal of the Meteorological Society of Japan. Ser. II, 83(5), 817-834.

970 Satyamurty, P. and Seluchi, M.E. (2007) Characteristics and structure of an upper air 971 cold vortex in the subtropics of South America. Meteorology and Atmospheric Physics 972 96(3-4), 203-220.

973 Scherhag, R. (1939) Der kälteeinbruch mitte Dezember 1938. Annalen Der 974 Hydrographie und Maritimen Meteorologie 67, 142.

975 Shapiro, M.A. (1970) On the applicability of the geostrophic approximation to upper976 level frontal-scale motions. Journal of the Atmospheric Sciences 27(3), 408-420.

977 Silva Dias, P.L., Schubert, W.H. and Demaria, M. (1983) Large-scale response of the 978 tropical atmosphere to transient convection. Journal of the Atmospheric Sciences 979 40(11), 2689-2707.

980 Simpson, R.H. (1952) Evolution of the Kona Storm, a subtropical cyclone. Journal of 981 Meteorology 9(1), 24-35. 
982 Sinclair, M.R. and Revell, M.J. (2000) Classification and composite diagnosis of 983 extratropical cyclogenesis events in the Southwest Pacific. Monthly Weather Review 984 128(4),1089-1105.

985 Singleton, A.T. and Reason, C.J.C. (2006) A Numerical model study of an intense 986 cutoff low pressure system over South Africa. Monthly Weather Review 135(3), 11289871150.

988 Stoelinga, M.T. (1996) A potential vorticity-based study of the role of diabatic heating 989 and friction in a numerically simulated baroclinic cyclone. Monthly Weather Review $990 \quad \mathbf{1 2 4}(5), 849-874$.

991 Trenberth, K.E. and Mo, K.C. (1985) Blocking in the Southern Hemisphere. Monthly 992 Weather Review 113(1), 3-21.

993 Wernli, H and Sprenger, M. (2007) Identification and ERA-15 climatology of potential 994 vorticity streamers and cutoffs near the extratropical tropopause. Journal of the 995 Atmospheric Sciences 64(5), 1569-1586.

996

997

998

999

1000

1001

1002

1003

1004 
1008

1009

1010

1011

1012

1013 Figure/Captions

1014

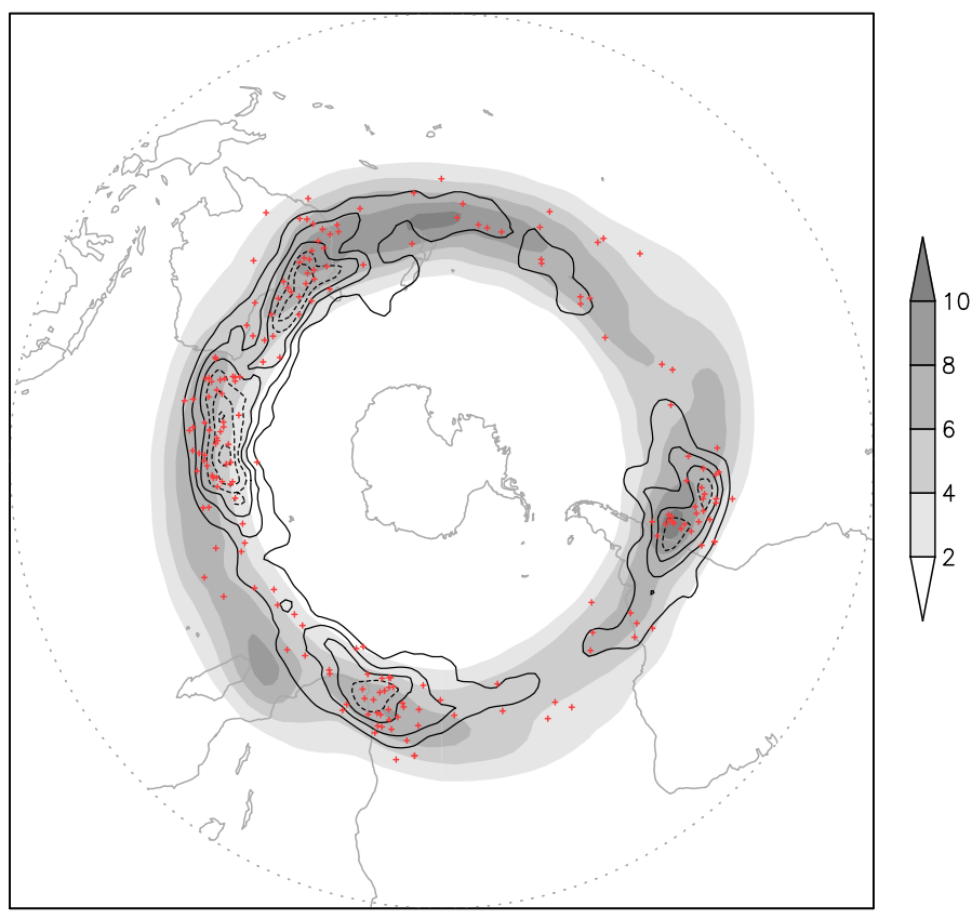

1015 Figure 1 Seasonal track density of the Southern Hemisphere Cut-off Lows obtained 1016 from the 200 strongest (black lines) and average (shaded) Cut-off Lows. The contour 1017 interval is 0.1 unit for density $\leq 0.3$ (solid line) and $\geq 0.4$ (dotted line). Symbol plus 1018 (red colour) denotes the locations of the maximum intensity (with respect to the $\xi_{300}$ in 
1019 each track for the strongest COLs. Analysis is performed using the Cut-off Lows that

1020 match between the $\xi_{300}$ and $Z_{300}^{\prime}$ from ERAI reanalysis for a 36-yr period (1979-2014).

1021 Unit is number per season per unit area, the unit area is equivalent to a $5^{\circ}$ spherical cap $1022\left(\cong 10^{6} \mathrm{~km}^{2}\right)$.

1023

1024

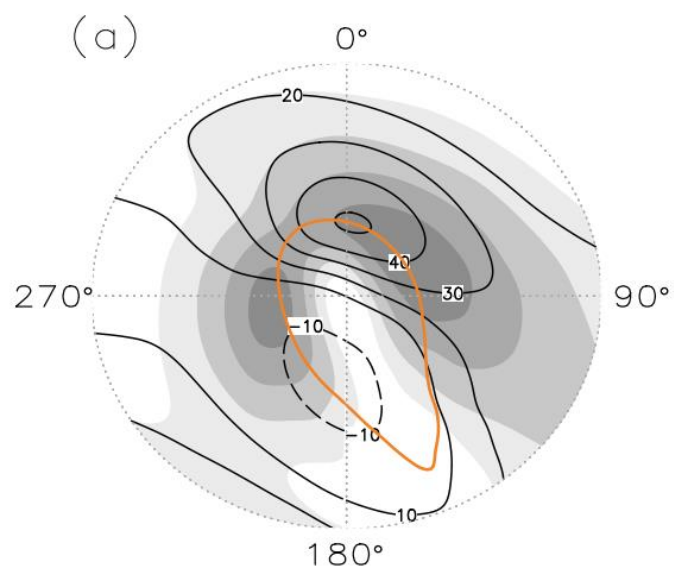

(c)

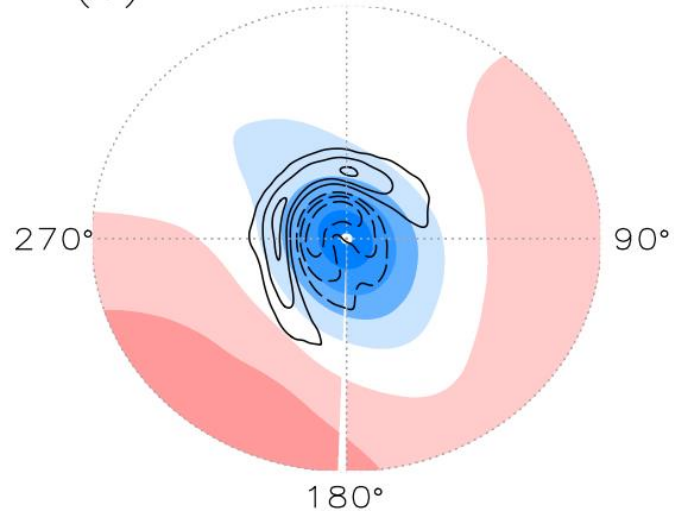

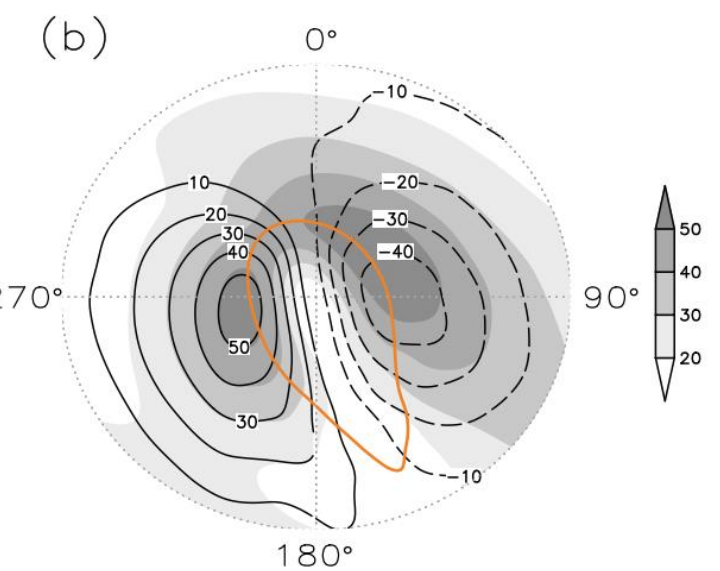

(d)

$0^{\circ}$

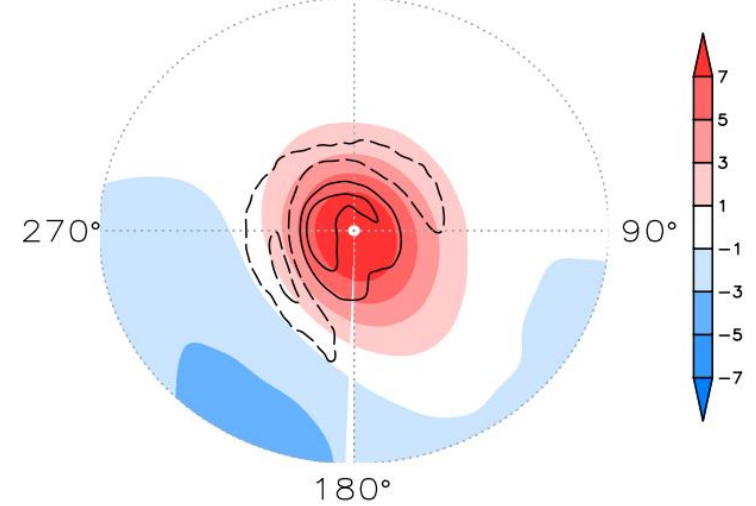

1025

Figure 2 Horizontal composites for the 200 most intense Cut-off Lows that match

1027 between the $\xi_{300}$ and $Z_{300}^{\prime}$ centred on the time and space relative to the $\xi_{300}$ minimum.

1028 Fields are: a) zonal wind and b) meridional wind (black line) combined with wind speed

1029 (shaded) in $\mathrm{m} / \mathrm{s}$, and PV for the $2 \mathrm{PVU}$ (orange line), all fields referred to $300 \mathrm{hPa}$; (c)

1030 and (d) are composites of temperature anomaly (shaded) in $\mathrm{K}$ and thermal frontal 
1031 parameter for contour intervals $0.4 \times 10^{-10} \mathrm{~K} /(100 \mathrm{~km})^{2}$ at (c) $400 \mathrm{hPa}$ and (d) $200 \mathrm{hPa}$.

1032 The distance from the centre of the COL composite to the edge is 15 degrees.

(a)

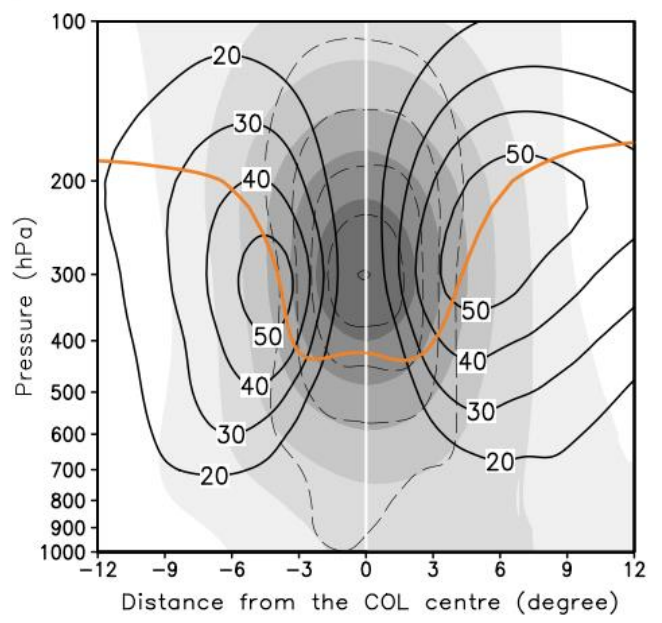

(c)

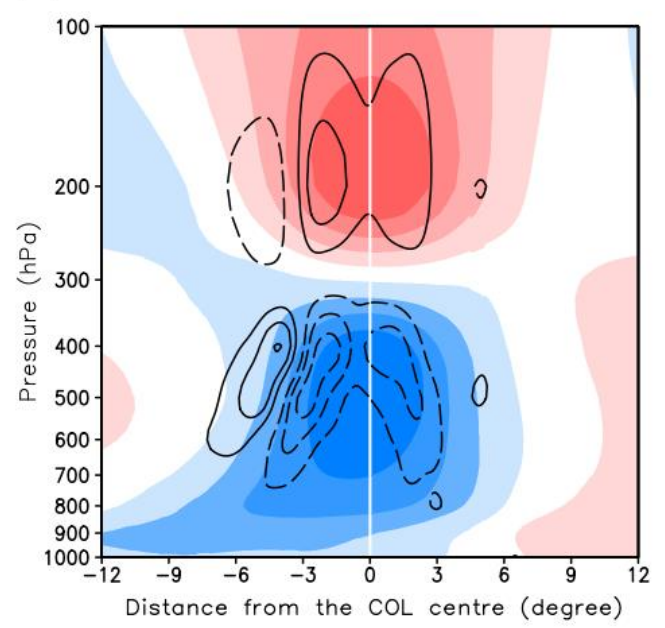

(b)

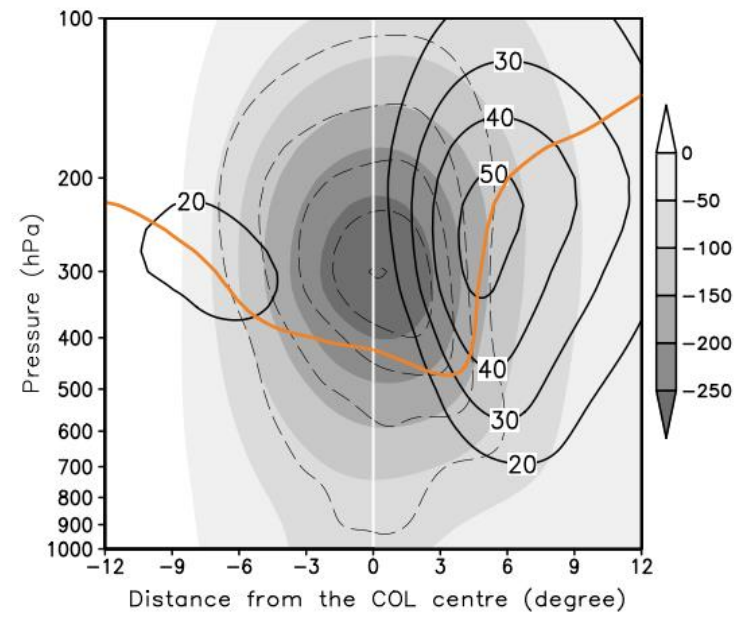

(d)

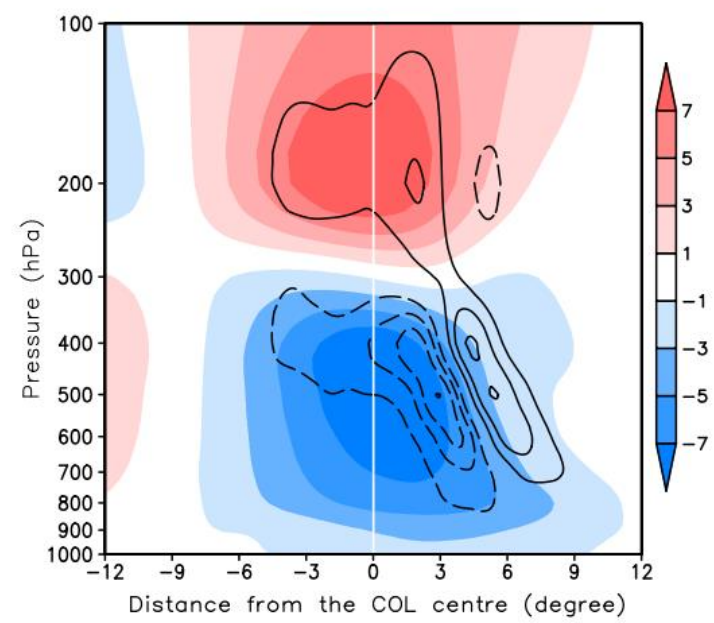

Figure 3 Composite vertical cross-sections along the (a),(c) west-east and (b),(d) southnorth lines of the 200 most intense Cut-off Lows that match between the $\xi_{300}$ and $Z_{300}^{\prime}$ centred on the time and space relative to the $\xi_{300}$ minimum. Fields are (a),(b) wind speed (solid line) in $\mathrm{m} / \mathrm{s}$, geopotential height anomaly (shaded) for contour intervals 50 gpm, relative vorticity (dashed lines) in $10^{-5} \mathrm{~s}^{-1}$ for contour intervals $3.0 \times 10^{-5} \mathrm{~s}^{-1}$; and PV for the 2 PVU (orange line); (c) (d) temperature anomaly (shaded) in K; and thermal 
1041 frontal parameter (black lines) in for contour intervals $0.4 \times 10^{-10} \mathrm{~K} /(100 \mathrm{~km})^{2}$, where 1042 solid (dashed) contours indicate positive (negative) values.

(a)

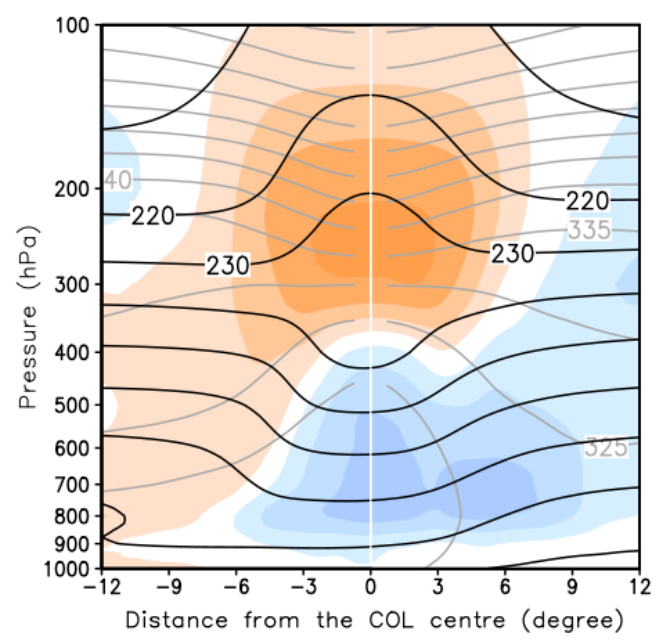

(c)

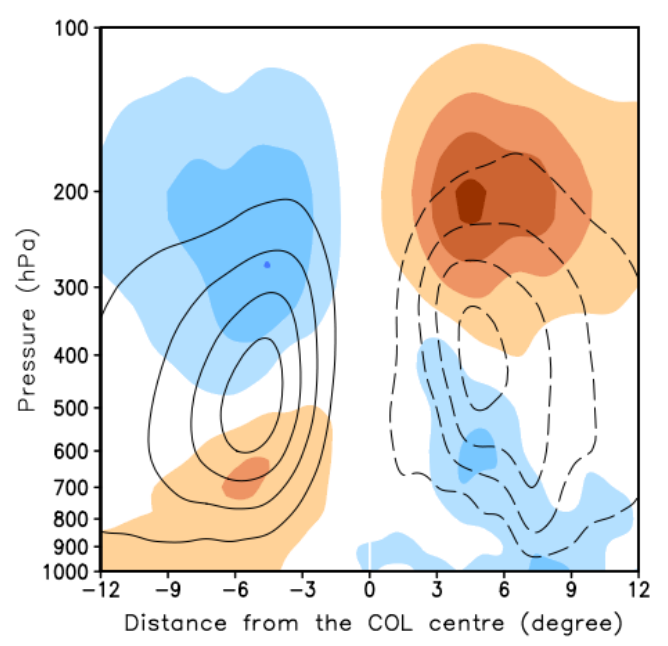

(b)

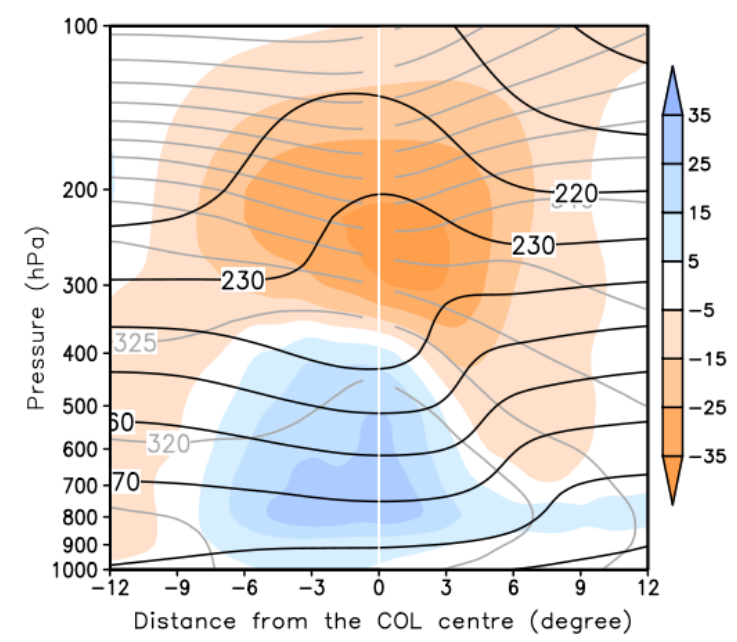

(d)

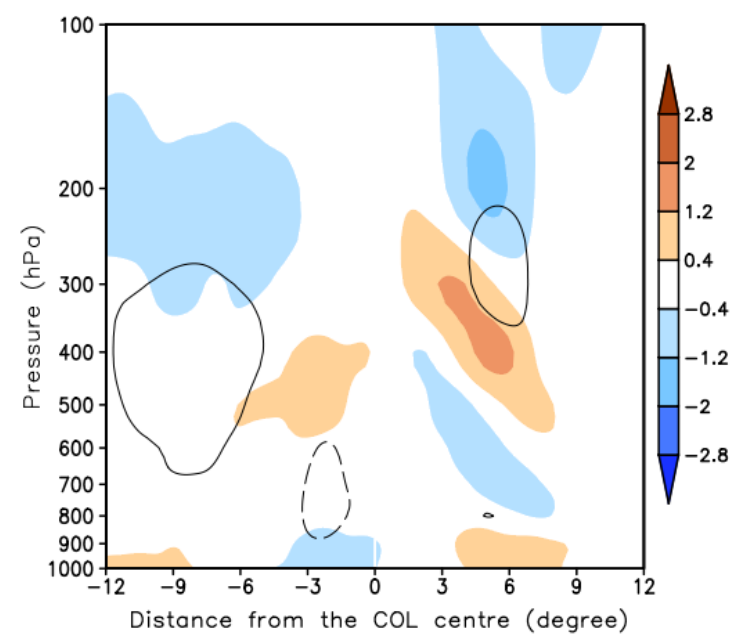

1046 Figure 4 Composite vertical cross-sections along the (a),(c) west-east and (b),(d) south-

1047 north lines of the 200 most intense Cut-off Lows that match between the $\xi_{300}$ and $Z_{300}^{\prime}$

1048 centred on the time and space relative to the $\xi_{300}$ minimum. Fields are (a),(b) relative

1049 humidity anomaly (shaded) in percentage, air temperature (black line) for contour

1050 intervals $10 \mathrm{~K}$, potential temperature (grey line) for contour intervals $5 \mathrm{~K}$, and (c),(d) 
1051 divergence (shaded) for contour intervals $0.810^{-5} \mathrm{~s}^{-1}$ and vertical velocity (black line) in contour intervals $0.1 \mathrm{~Pa} \mathrm{~s}^{-1}$, where solid (dashed) contours indicate positive (negative) 1053 values.

(a)
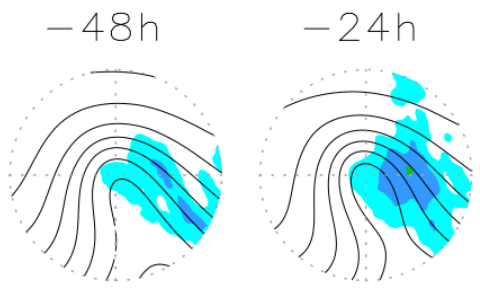

(b)

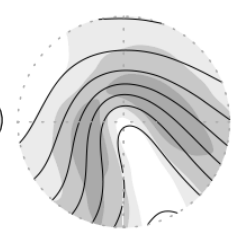

(c)

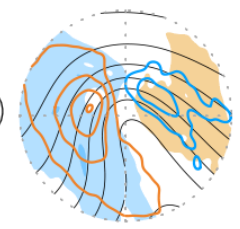

(d)
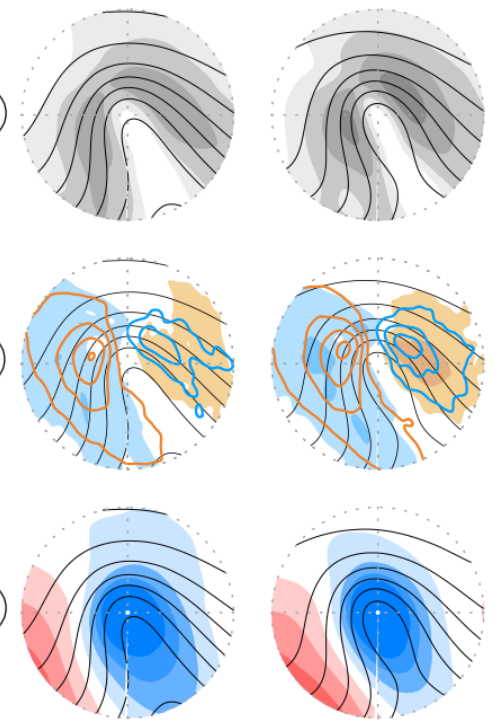
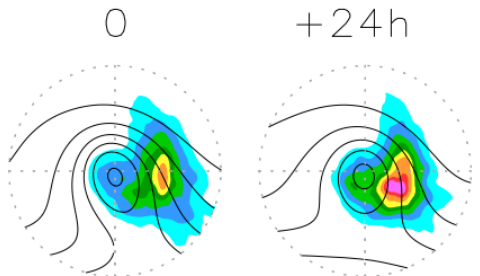

$+48 h$
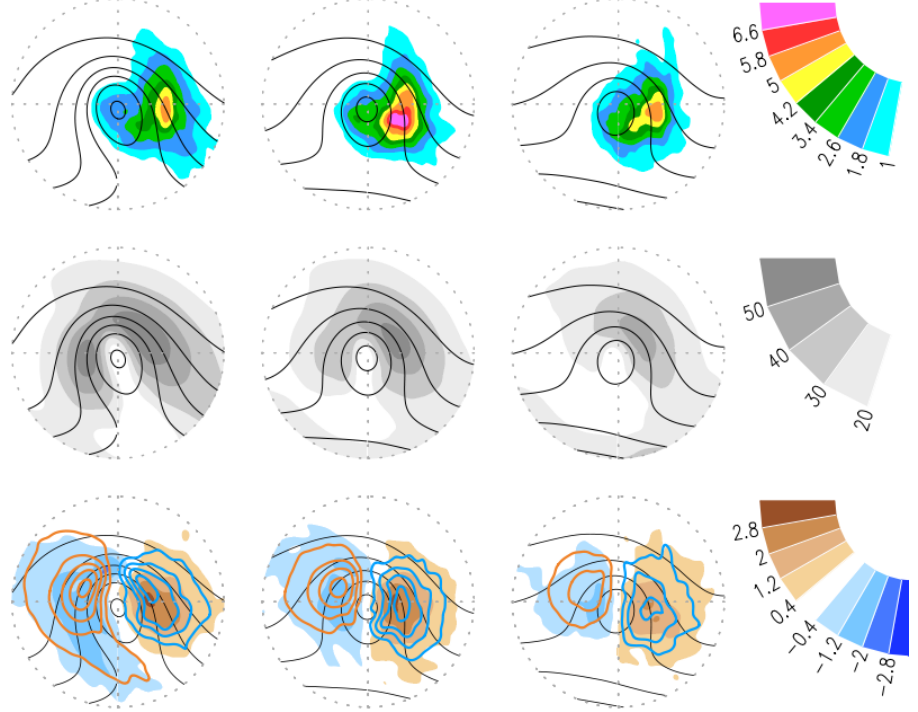

1054
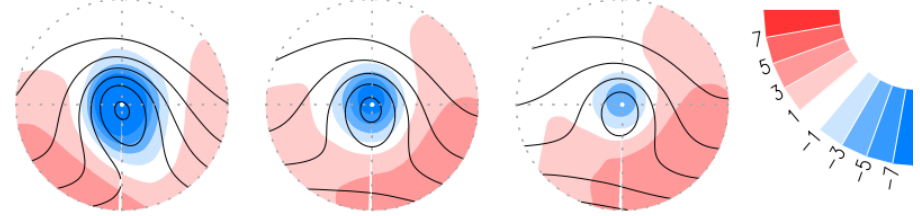

1055

Figure 5 Life cycle composite of the Southern Hemisphere Cut-off Lows for the period between two days before and two days after the time of maximum intensity in $\xi_{300}$ (day zero). Fields are: (a) 6-hourly accumulated precipitation in mm (shaded); (b) absolute wind speed in $\mathrm{m} / \mathrm{s}$ (shaded); (c) divergence in $10^{-5} \mathrm{~s}^{-1}$ (shaded) and vertical velocity for contour intervals $0.1 \mathrm{~Pa} \mathrm{~s}^{-1}$ for positive (negative) values in orange (blue) colour; and

1060 (d) temperature anomaly in $\mathrm{K}$ (shaded). All fields are combined with the $Z_{300}$ height for 1 b61 contour intervals 100 gpm (black contour). 


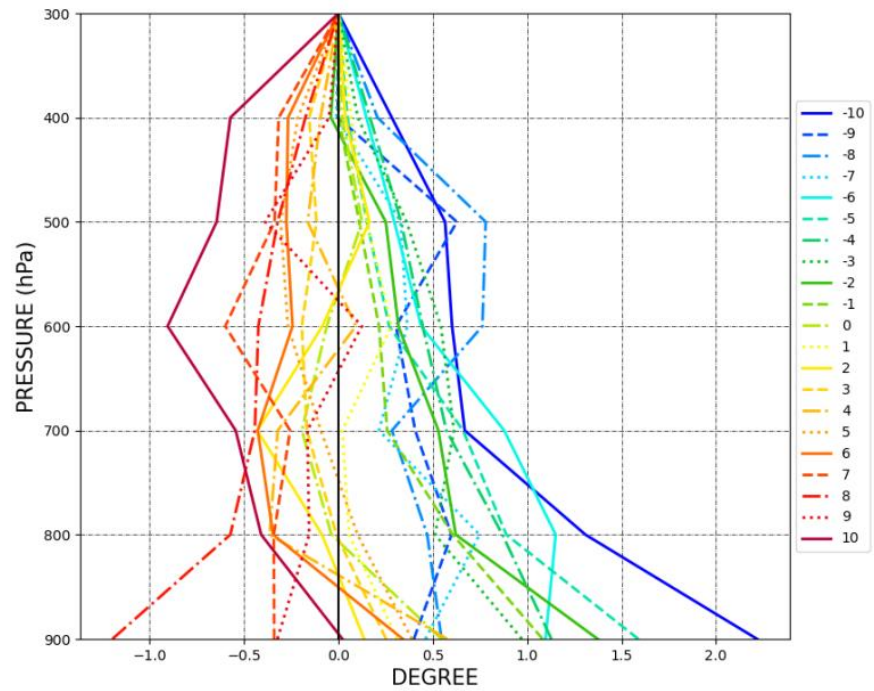

1064 Figure 6 Vertical tilt life cycle composite for the Southern Hemisphere Cut-off Lows.

1065 Composite is obtained using the $\xi_{300}$ Cut-off Lows. The time steps are for 6-hour 1066 interval, shown up to $60 \mathrm{~h}$ on either side of the $\xi_{300}$ minimum (time zero). Tilts are in 1067 geodesic angle from the $\xi_{300}$ minimum. 


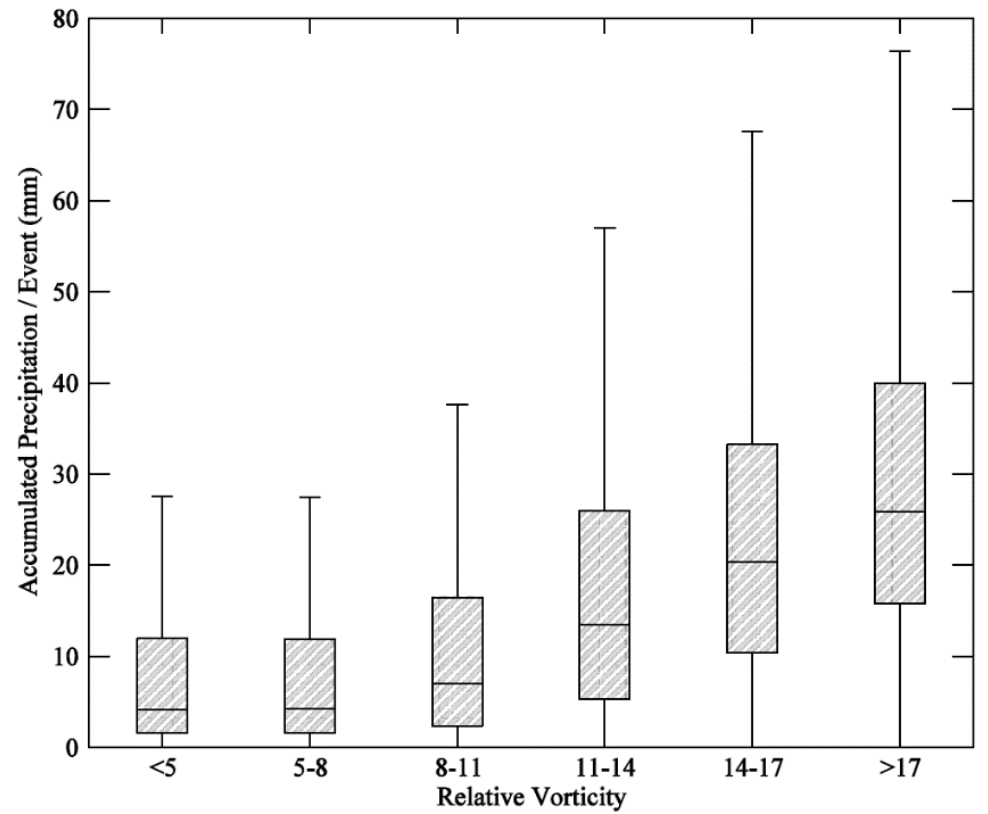

1074 Figure 7 Boxplot illustrating the cumulative precipitation (mm) along the tracks of all 1075 identified Cut-off Lows with respect to intensity range. Intensity is measured in terms of 1076 the maximum $\xi_{300}$ scale by $-1.0 \times 10^{-5} \mathrm{~s}^{-1}$. The number of identified Cut-off Lows for 1077 each intensity range is: $1,084(<5.0) ; 3,738(5.0-8.0)$; 5,360 (8-11); 4,856 (11.0-14.0); $10782,693(14.0-17.0) ; 955(>17.0)$. The top and bottom lines of the light gray box denote 1079 the 75th and 25th percentiles, respectively; the black line in the box centre represents 1080 the 50th percentile (median); the top and bottom whiskers indicate the upper and lower 1081 extremes, respectively. 

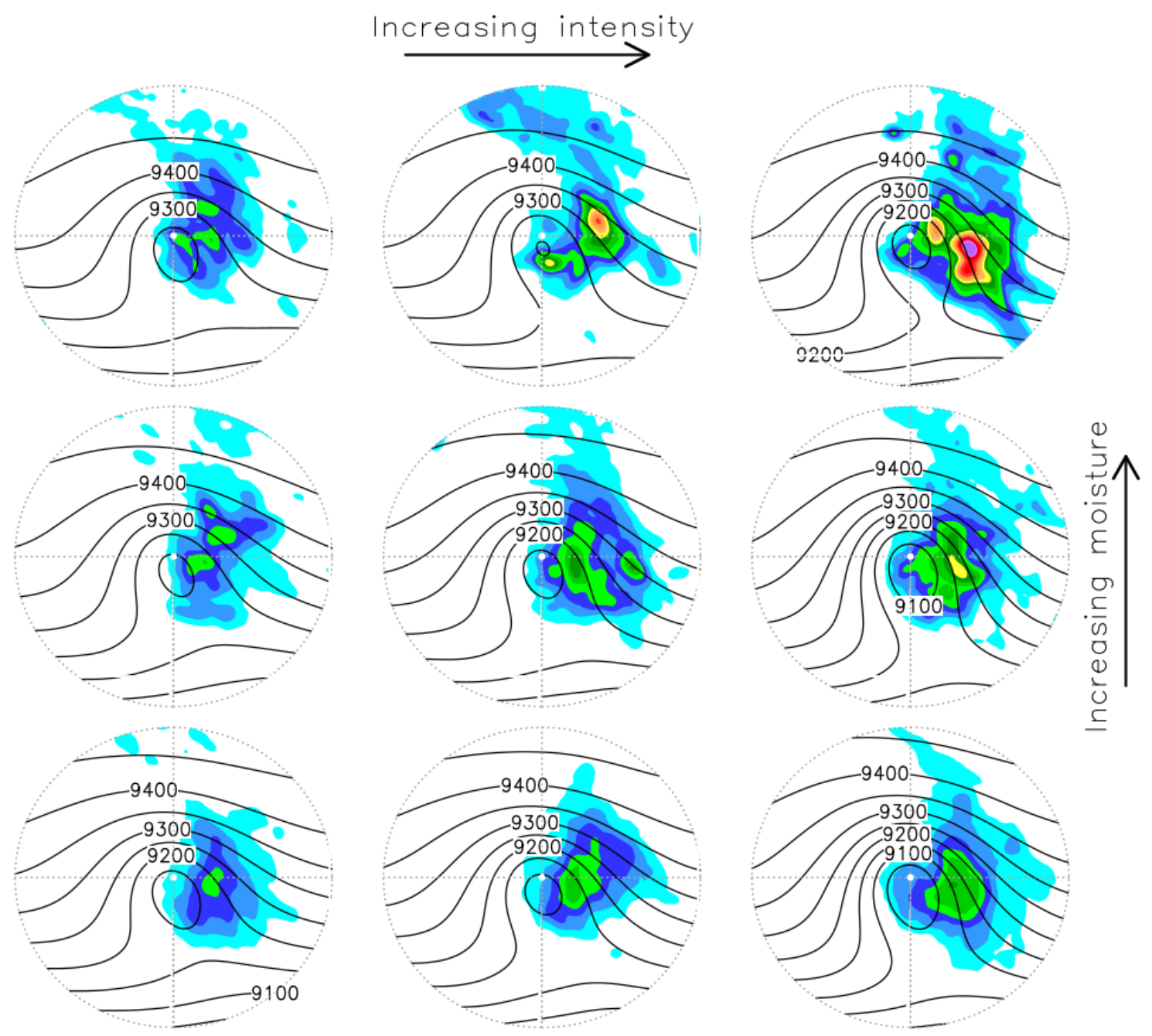

1082
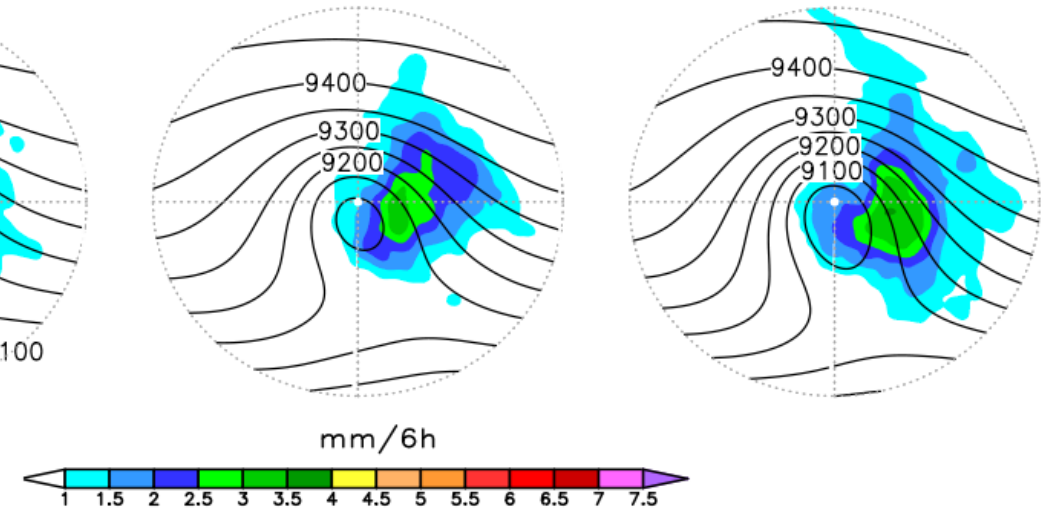

1083 Figure 8 Composites of Southern Hemisphere Cut-off Lows for precipitation as a

1084 function of intensity and moisture. Plots are precipitation in $\mathrm{mm} / 6 \mathrm{~h}$ (shaded) and $Z_{300}$

1085 in gpm (solid line). Composites are determined as a function of intensity $\left(\xi_{300}\right.$,

1086 increasing from left to right) and moisture (total column water, increasing from bottom

1087 to top). The two fields are calculated using the $\xi_{300}$ Cut-off Lows for the time of $\xi_{300}$

1088 minimum. Precipitation is calculated using area average within $5^{\circ}$ spherical arc radius

1089 centred on the $\xi_{300}$ minimum. The categories are: [intensity]: 9.3-10.5, 10.5-12.0, 12.0-

$109019.0 \mathrm{~s}^{-1}$ (scaled by $-1 \times 10^{-5}$ ); and [moisture]: $27.6-30.8,30.8-34.7,34.7-40.0 \mathrm{~kg} / \mathrm{m}^{2}$. The

1091 number of Cut-off Lows used in each nine composite and the corresponding area- 
1092 Figure 8 (continuation) average accumulated precipitation (mm) over the whole 1093 lifecycle are, respectively: top left $(126 ; 13.8)$, top centre $(73 ; 15.6)$, top right $(49 ; 20.1)$, 1094 middle left $(145 ; 15.0)$, middle centre $(135 ; 18.7)$, middle right $(91 ; 21.6)$, bottom left $1095 \quad(207 ; 18.7)$, bottom centre $(182 ; 20.9)$, bottom right $(169 ; 26.1)$.

a)

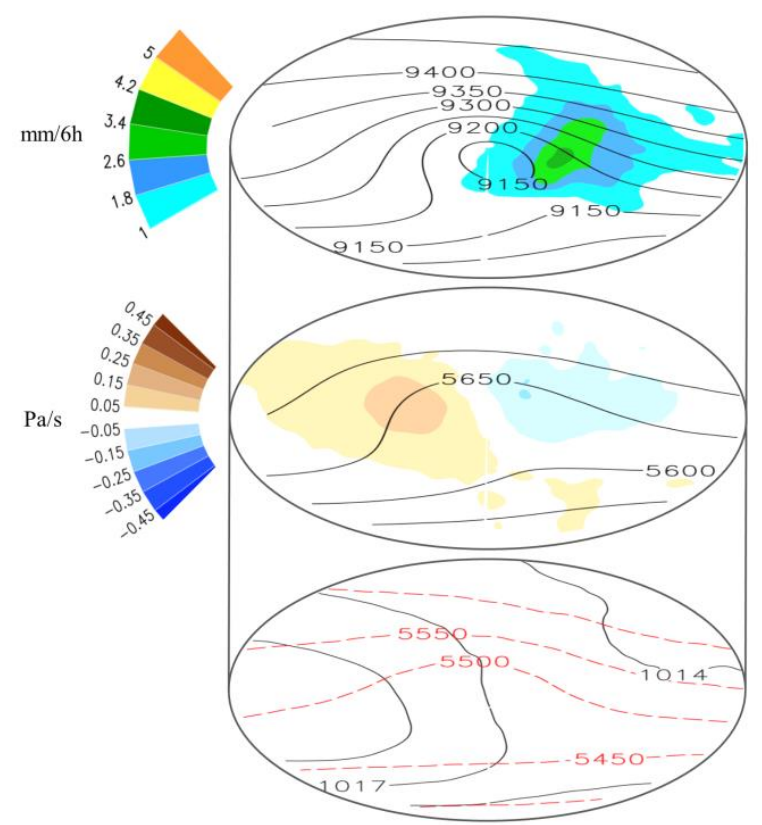

b)

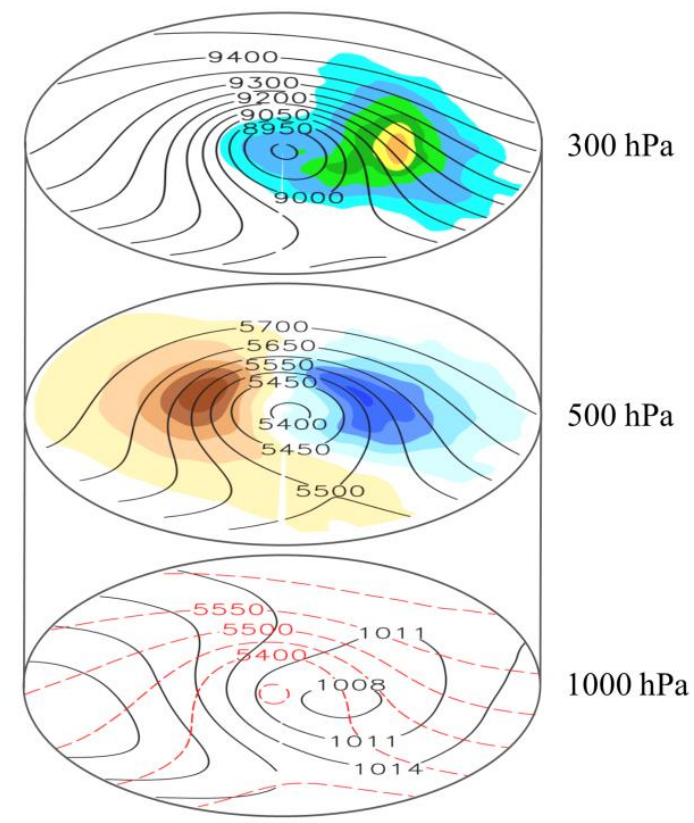

1097

1098 Figure 9 A three-dimensional schematic view of the a) average Cut-off Lows and the b)

1099 strongest Cut-off Lows. Fields are: [top]: 300-hPa geopotential height (black line) in

1100 gpm and precipitation (shaded) in $\mathrm{mm} / 6 \mathrm{~h}$; [middle]: 500-hPa geopotential height (black

1101 line) in gpm and 500-hPa vertical velocity (shaded) in $\mathrm{Pa} \mathrm{s}^{-1}$; [bottom]: mean sea level

1102 pressure (black line) in $\mathrm{hPa}$ and 1000-500 hPa thickness (red dashed line) in gpm.

1103 Analysis performed using the Cut-off Lows that match between the $\xi_{300}$ and $Z_{300 \text {. }}^{\prime}$ 


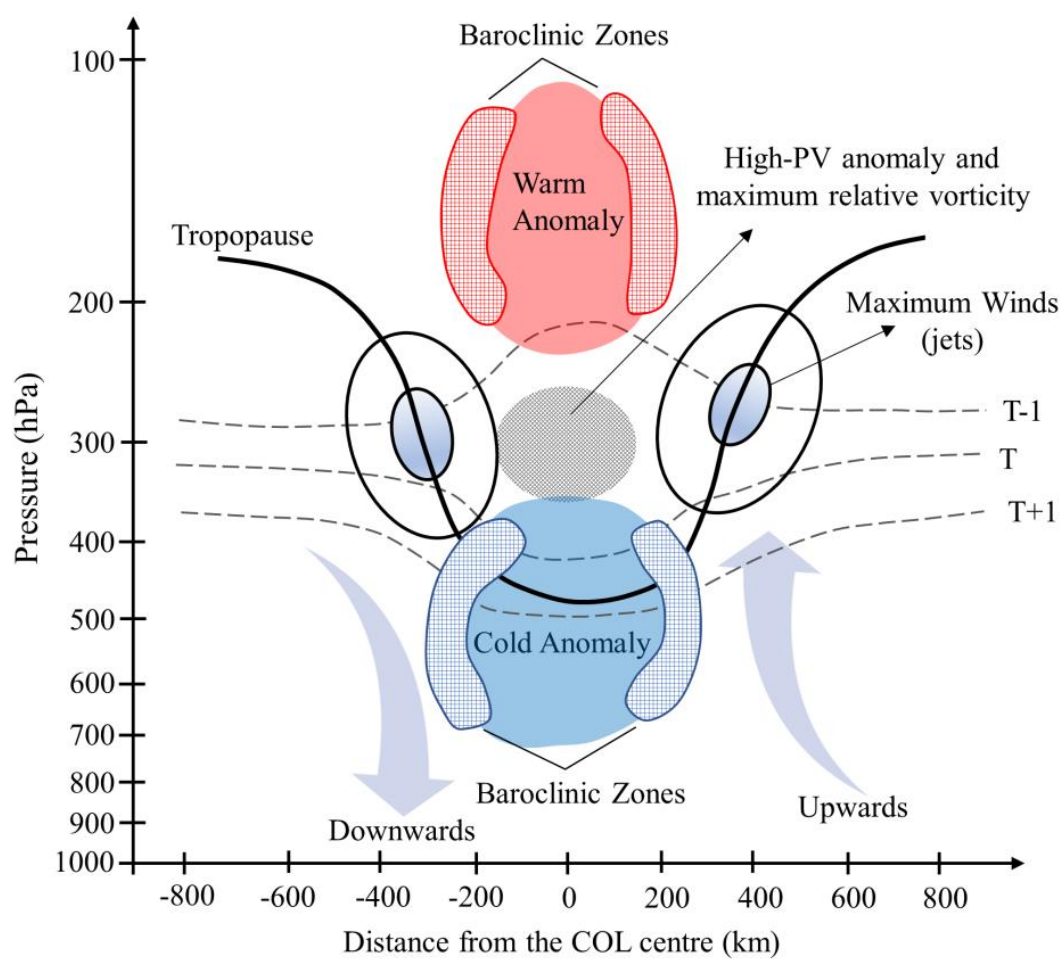

1104

1105 Figure 10 Schematic of typical structural features during the mature stage of a Cut-off

1106 Low (west-east cross-section). The thick black line represents the dynamical tropopause

1107 (2.0 PVU surface), gray dashed line indicates temperature, wide arrows indicate the

1108 vertical motions, the blue and red colour regions mean the cold and warm anomalies

1109 respectively with their associated baroclinic zones (in texture). The gray texture

1110 indicates the high-PV anomaly and maximum magnitude of relative vorticity. Adapted

$1 \mid 11$ from Llasat et al. (2006). 